Document downloaded from:

http://hdl.handle.net/10251/56506

This paper must be cited as:

Puerto Albandoz, J.; Tamir, A.; Perea Rojas Marcos, F. (2011). A cooperative location game based on the 1-center location problem. European Journal of Operational Research. 214(2):317-330. doi:10.1016/j.ejor.2011.04.020.

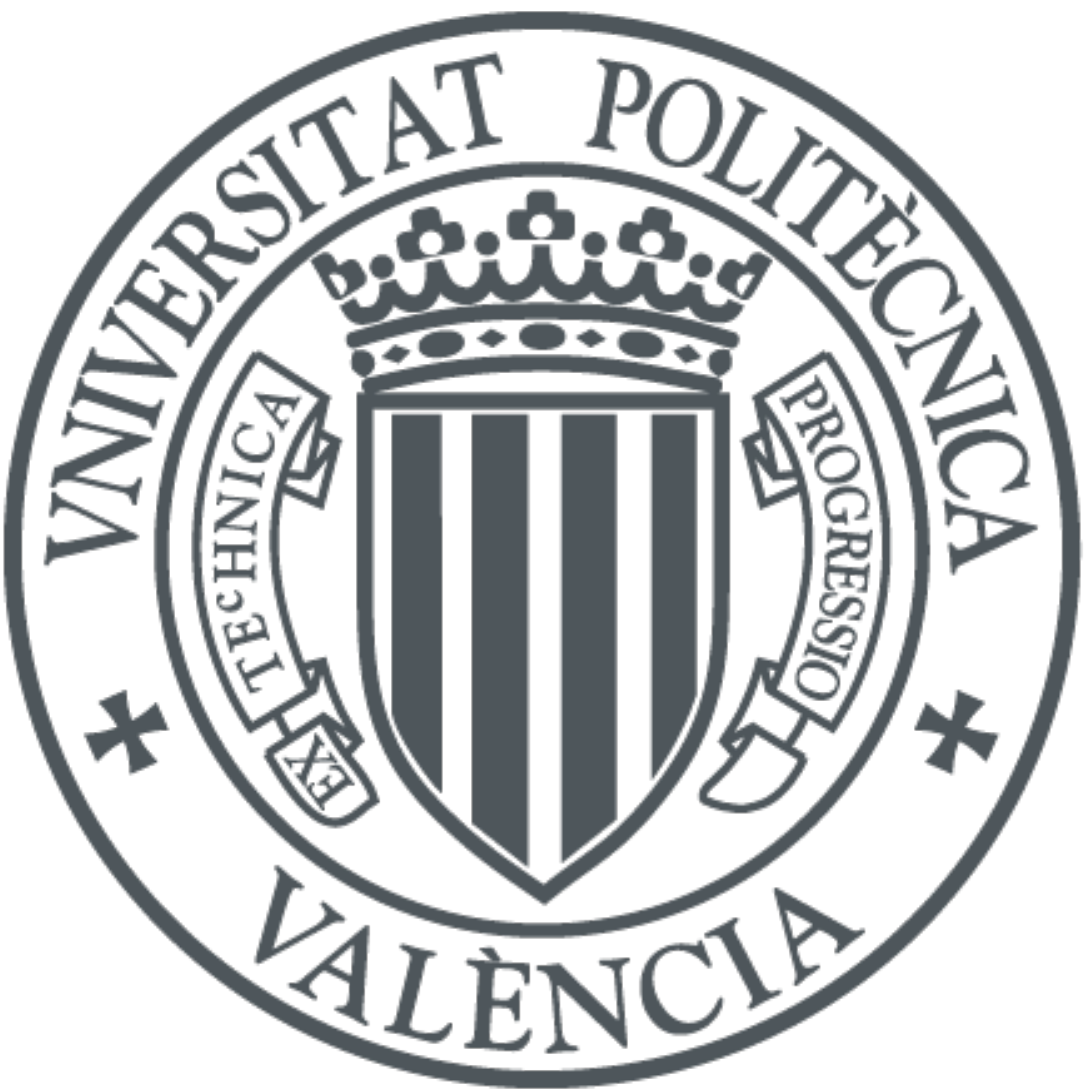

The final publication is available at

http://dx.doi.org/10.1016/j.ejor.2011.04.020

Copyright Elsevier

Additional Information 


\title{
A Cooperative Location Game Based on the 1-Center Location Problem
}

\author{
Justo Puerto \\ Facultad de Matemáticas \\ Universidad de Sevilla, Spain \\ Arie Tamir \\ School of Mathematical Sciences \\ Tel Aviv University, Israel \\ Federico Perea \\ Departamento de Estadística e Investigación Operativa Aplicadas y Calidad \\ Universidad Politécnica de Valencia, Spain
}

February 4, 2011

\begin{abstract}
In this paper we introduce and analyze new classes of cooperative games related to facility location models defined on general metric spaces. The players are the customers (demand points) in the location problem and the characteristic value of a coalition is the cost of serving its members. Specifically, the cost in our games is the service radius of the coalition.

We study the existence of core allocations and the existence of polynomial representations of the cores of these games, focusing on network spaces, i.e., finite metric spaces induced by undirected graphs and positive edge lengths, and on the $\ell_{p}$ metric spaces defined over $\mathbb{R}^{\mathrm{d}}$.
\end{abstract}

Keywords: Cooperative combinatorial games, core solutions, radius, diameter.

\section{Introduction}

Let $X$ be a metric space and let $N_{0}=\left\{v_{0}, v_{1}, \ldots, v_{k}\right\}$ be a finite set of points in $X$. The subset $N=\left\{v_{1}, \ldots, v_{k}\right\}$ is identified as the set of $k$ players, and we refer to these points as existing facilities, or demand points. There is also a distinguished point $v_{0}$, representing the location of a server that provides services to the players, that can be viewed as an essential element in the system, e.g., each demand point must have access to $v_{0}$. Note that $v_{0}$ is not a player. For motivation purposes, assume that the demand points represent patients, and $v_{0}$ 
is the location of a repairman or a medical doctor who provides assistance or health services, respectively.

Our study is motivated by location models, where the time elapsed till the service is provided (response time) is critical. Moreover, referring to the above example, the service of the doctor is not necessarily provided at his home base. Instead, the coalition of patients $S$ can optimally select the location of the center, (e.g., clinic or hospital), where the service will be provided. When there is a call for service, both, a patient and the doctor will travel to the clinic. The cost of service is assumed to be the service radius, defined as the maximum distance travelled by a patient or the doctor to the service facility (center). Using location theory terminology, the cost of a coalition $S$ is the solution value of the 1-center problem for the set $S \cup\left\{v_{0}\right\}$.

In the rest of the paper we will use the concepts of diameter and radius. Given a finite subset of points $Y \subseteq X$, its diameter $D(Y)$, is defined by

$$
D(Y)=\max _{y_{1}, y_{2} \in Y} d\left(y_{1}, y_{2}\right)
$$

A pair of points $y_{1}, y_{2} \in Y$, satisfying $D(Y)=d\left(y_{1}, y_{2}\right)$ is called a diametrical pair. The radius of $Y$ is defined by

$$
R(Y)=\inf _{x \in X} \max _{y \in Y} d(x, y) .
$$

A point $x \in X$ satisfying $R(Y)=\max _{y \in Y} d(x, y)$ is called a 1-center of $Y$. Note that by the triangle inequality

$$
R(Y) \leq D(Y) \leq 2 R(Y)
$$

We now formally define the class of cooperative cost games based on the above facility location problems that we study in this paper: The Minimum Radius Location Game (MRLG).

First recall that a generic finite cooperative game is a pair $(N, v)$, where $N$ is a finite set of players and $v$ is the characteristic function defined from $2^{N}$ to $\mathbb{R}$, which satisfies $v(\emptyset)=0$, and assigns to each coalition $S \subseteq N$ a real value (it can be a benefit or a cost). The game $(N, v)$ is called monotone if for any pair of subsets $S_{1} \subseteq S_{2} \subseteq N, v\left(S_{1}\right) \leq v\left(S_{2}\right)$. It is called subadditive if for any pair of subsets $S_{1}, S_{2} \subseteq N, v\left(s_{1} \cup S_{2}\right) \leq v\left(S_{1}\right)+v\left(S_{2}\right)$, and it is called submodular if for any pair of subsets $S_{1}, S_{2} \subseteq N, v\left(s_{1} \cup S_{2}\right)+v\left(S_{1} \cap S_{2}\right) \leq v\left(S_{1}\right)+v\left(S_{2}\right)$.

The core of $(N, v)$ (in the case of a cost game) is the set

$$
C(N, v)=\left\{x \in \mathbb{R}^{k}: x(N)=v(N), x(S) \leq v(S), \forall S \subseteq N\right\},
$$

where $x(S)=\sum_{j: v_{j} \in S} x_{j}$, for any $S \subseteq N$.

The first game, which we have studied in a companion paper, Puerto et al. (2010), is the 
Minimum Diameter Location Game (MDLG), $\left(N, v_{I}\right)$, with respect to the metric space $X$ and the set of points $N_{0}$. Its characteristic function is defined by

$$
v_{I}(S)=D\left(S \cup\left\{v_{0}\right\}\right)
$$

The second game, which we study in this paper, is the Minimum Radius Location Game (MRLG), $\left(N, v_{I I}\right)$, with respect to the metric space $X$ and the set of points $N_{0}$. Its characteristic function is defined by

$$
v_{I I}(S)=2 R\left(S \cup\left\{v_{0}\right\}\right) .
$$

(The factor 2 in the above definition is used for convenience and comparison purposes only.)

It directly follows from the definitions that both games are monotone. Also, from (1), for any $S \subseteq N$,

$$
v_{I}(S) \leq v_{I I}(S) \leq 2 v_{I}(S)
$$

In our companion paper we have shown that $C\left(N, v_{I}\right)$, the core of the MDLG, $\left(N, v_{I}\right)$, is always nonempty. Moreover, there is a vector in $C\left(N, v_{I}\right)$ where at most 2 of its components are positive and the rest are zero. We have also proved that recognizing whether a given vector $x$ is in $C\left(N, v_{I}\right)$ is NP-hard.

In contrast, in this paper we will demonstrate that $C\left(N, v_{I I}\right)$, the core of the MRLG, can be empty. In view of this result we will prove that for several important metric spaces the core, which by definition is a polyhedral set in $\mathbb{R}^{k}$, is nonempty and/or has a polyhedral representation by $O\left(k^{c}\right)$ linear inequalities ( $c$ is independent of the number of players $k$, and depends only on some parameters of the space $X$.) Such a representation is usually called efficient or compact. One of these metric spaces is the network metric space induced by a connected undirected graph and its positive edge lengths. It is defined as follows:

Suppose $G=(V, E)$ is a connected undirected graph with positive edge lengths $\left\{l_{e}\right\}, e \in$ $E$, where $V=\left\{v_{0}, v_{1}, \ldots, v_{n}\right\}$. When $e=\left(v_{i}, v_{j}\right)$, we will also use the notation $l\left(v_{i}, v_{j}\right)=l_{e}$. Each edge in $E$ is assumed to be rectifiable. We refer to interior points on an edge by their distances (along the edge) from the two nodes of the edge. $A(G)$ is the continuum set of points on the edges of $G$. For any pair of points $x, y \in A(G)$, we let $d(x, y)$ denote the length of a shortest path in $A(G)$ connecting $x$ and $y$. We refer to $A(G)$ as the metric space induced by $G$ and the edge lengths.

The paper is structured as follows. In Section 2, we demonstrate that in general the core of the MRLG can be empty, even for a geometric planar road network where the edges are straight lines and their lengths are the respective Euclidean distances. We observe that for discrete spaces the MRLG may not even be subadditive, and we then prove that in the case of geodesic spaces it is always subadditive. We also provide sufficient conditions for the existence of core allocations, based on the relationship between the MRLG and the MDLG. 
This relationship is then used to show that even when the core of the MRLG may be empty, any core allocation of the MDLG is a $\{1 / 2\}$-budget balanced allocation of the MRLG.

Section 3 is devoted to network metric spaces $A(G)$. For such spaces we assume without loss of generality that the set of players $N$ is a subset of $V$. When $N=V \backslash\left\{v_{0}\right\}$ we call the game a complete game. We present two interesting classes of graphs for which the core of the MRLG is nonempty: median and long-diameter graphs. For any graph $G$, we provide a representation of $C\left(N, v_{I I}\right)$ by $O\left(m|N|^{2}\right)$ linear constraints, where $m$ is the number of edges of $G$. Such a representation implies that emptiness of the core can be efficiently checked with linear programming methods.

A similar efficient representation having $O\left(|X|^{2}\right)$ constraints for a general discrete metric space $X$, is given in Section 4. The special case in which $N=X-\left\{v_{0}\right\}$ will be called a complete discrete game.

In Section 5 we study the case in which the underlying space is the $\ell_{p}$ metric space over $\mathbb{R}^{\mathrm{d}}$, and give an efficient representation of $C\left(N, v_{I I}\right)$, for the case where the dimension $\mathrm{d}$ is fixed. We show that in the case of the infinity norm the core is always nonempty. In the case of other norms, the emptiness is still an open question, with the exception of the planar Euclidean case. For the latter case, which is of great importance from the application point of view, we constructively generate a core allocation where the total cost is assigned to the (at most) three demand points defining the smallest circle enclosing all the demand points and the service point. The proof of this result is given in the Appendix. (This is in contrast with the example in Section 2 which shows that the core can be empty for a geometric planar road network where the edges are straight lines and their lengths are the respective Euclidean distance.)

The paper ends with some conclusions and open problems. Specifically, it is still unclear what general properties of the radius game will at least unify all the non-emptiness results presented in this paper. Table 1 summarizes the main results in the paper.

\section{Emptiness of the core $C\left(N, v_{I I}\right)$}

We have already noted that by definition the characteristic function $v_{I I}$ is monotone. However, when the metric space $X$ is discrete, i.e., $|X|$ is finite, the radius location game, $\left(N, v_{I I}\right)$ may not exhibit the subadditivity property. As a result players may have no incentive to cooperate and the core can be empty, as shown in the next example.

Example 2.1 Consider a 5-node path with edge set $E=\left\{\left(v_{1}, v_{2}\right),\left(v_{2}, v_{0}\right),\left(v_{0}, v_{3}\right),\left(v_{3}, v_{4}\right)\right\}$. The respective edge lengths are 1,1,2 and 2, as shown in Figure 1.

The finite (discrete) space $X$ consists of the 5 nodes (points) with the distance function induced by the edge lengths. $X$ can also be viewed as a set of 5 points on the real line. 


\begin{tabular}{|c|c|c|c|}
\hline & & $\begin{array}{c}\text { Complete } \\
\text { Radius Game }\end{array}$ & Radius Game \\
\hline \multicolumn{2}{|c|}{ Discrete Spaces } & $\begin{array}{c}\text { Empty } \\
\text { (3-players) }\end{array}$ & $\begin{array}{c}\text { Empty (2-players) } \\
\text { Core's polynomial representation }\end{array}$ \\
\hline \multirow{7}{*}{$\begin{array}{l}\text { Spaces } \\
\text { with } \\
\text { continuum }\end{array}$} & General & & $\begin{array}{c}\text { Empty } \\
(3 \text {-players }) \\
\end{array}$ \\
\hline & $\begin{array}{l}\text { Space } \\
A(G)\end{array}$ & $\begin{array}{c}\text { Open } \\
\text { (nonempty } \\
\text { 3-players) }\end{array}$ & $\begin{array}{c}\text { Empty } \\
\text { (3-players) } \\
\text { Core's polynomial representation }\end{array}$ \\
\hline & $\begin{array}{l}\text { Space } \\
A(T)\end{array}$ & $\begin{array}{c}\text { Nonempty } \\
\text { (submodular) }\end{array}$ & $\begin{array}{c}\text { Nonempty } \\
\text { (submodular) } \\
\text { Core's polvnomial representation }\end{array}$ \\
\hline & $\left(\mathbb{R}^{\mathrm{d}}, \ell_{p}\right)$ & & Core's polynomial representation \\
\hline & $\left(\mathbb{R}^{\mathrm{d}}, \ell_{1}\right)$ & & $\begin{array}{c}\text { nonempty, } \mathrm{d}=1,2 \\
\text { open, } \mathrm{d} \geq 3 \\
\text { Core's polynomial representation }\end{array}$ \\
\hline & $\left(\mathbb{R}^{\mathrm{d}}, \ell_{2}\right)$ & & $\begin{array}{c}\text { nonempty, } \mathrm{d}=1,2 \\
\text { open, } \mathrm{d} \geq 3 \\
\text { Core's polynomial representation }\end{array}$ \\
\hline & $\left(\mathbb{R}^{\mathrm{d}}, \ell_{\infty}\right)$ & & $\begin{array}{c}\text { nonempty, } \mathrm{d} \geq 1 \\
(2 \text { players pay }) \\
\text { Core's polynomial representation }\end{array}$ \\
\hline
\end{tabular}

Table 1: Summary of results on the core $C\left(N, v_{I I}\right)$.

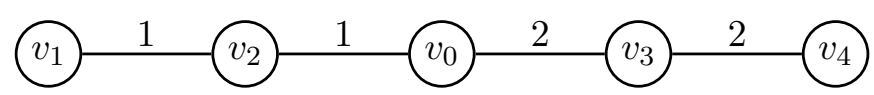

Figure 1: Graph in Example 2.1.

Consider first the 2-player game on $X$ defined by $N=\left\{v_{1}, v_{4}\right\}$. It is not subadditive since $v_{I I}\left(\left\{v_{1}, v_{4}\right\}\right)>v_{I I}\left(\left\{v_{1}\right\}\right)+v_{I I}\left(\left\{v_{4}\right\}\right)$.

The above example can easily be modified to show that subadditivity may not hold even for complete discrete games, i.e., when $N=X \backslash\left\{v_{0}\right\}$. Specifically, consider the complete 4-player radius game defined on the above set $X$, and let $N=X \backslash\left\{v_{0}\right\}=\left\{v_{1}, v_{2}, v_{3}, v_{4}\right\}$.

The smallest discrete neighborhood covering all nodes has radius 4 , while the smallest (discrete) neighborhoods covering $\left\{v_{1}, v_{2}, v_{0}\right\}$ and $\left\{v_{3}, v_{4}, v_{0}\right\}$ have radii 1 and 2 , respectively. Hence, $v_{I I}\left(\left\{v_{1}, v_{2}, v_{3}, v_{4}\right\}\right)=8, v_{I I}\left(\left\{v_{1}, v_{2}\right\}\right)=2, v_{I I}\left(\left\{v_{3}, v_{4}\right\}\right)=4$, and therefore $v_{I I}\left(\left\{v_{1}, v_{2}, v_{3}, v_{4}\right\}\right)>v_{I I}\left(\left\{v_{1}, v_{2}\right\}\right)+v_{I I}\left(\left\{v_{3}, v_{4}\right\}\right)$.

It is easy to check that unlike the above 2-player radius game defined on a discrete metric space, every complete 2-player game, defined on a 3 point discrete metric space has a nonempty core. The last example illustrates that a complete 4-player radius game, defined on a 5 point discrete metric space may not be subadditive, and therefore can have an empty 
core. The next example shows that a complete 3-player game, defined on a 4 point discrete metric space may be subadditive, and still has an empty core.

Example 2.2 Consider the discrete space defined by $X=\left\{v_{0}, v_{1}, v_{2}, v_{3}\right\}, d\left(v_{0}, v_{1}\right)=d\left(v_{2}, v_{3}\right)$ $=2$ and $d\left(v_{0}, v_{2}\right)=d\left(v_{0}, v_{3}\right)=d\left(v_{1}, v_{2}\right)=d\left(v_{1}, v_{3}\right)=1$. Let $N=\left\{v_{1}, v_{2}, v_{3}\right\}$, and consider the (discrete) radius game $\left(N, v_{I I}\right)$. We have $v_{I I}(N)=4$ and $v_{I I}(S)=2$, for any coalition $S$, with $|S| \leq 2$. It is easy to see that there is no vector $x=\left(x_{1}, x_{2}, x_{3}\right)$ satisfying $x_{1}+x_{2}+x_{3}=4$, $x_{1}+x_{2} \leq 2, x_{2}+x_{3} \leq 2$, and $x_{1}+x_{3} \leq 2$.

When the metric space $X$ consists of a continuum set of points $C\left(N, v_{I I}\right)$ can also be empty for a 3-player game, as illustrated by the next example of a network metric space $A(G)$. This example corresponds to a very simple geometric planar road network, where the edges are line segments and their lengths are the respective Euclidean distances.

Example 2.3 Consider the graph $G=(V, E)$ where $V=\left\{v_{0}, v_{1}, \ldots, v_{6}\right\}$ and $E=\left\{\left(v_{0}, v_{4}\right)\right.$, $\left.\left(v_{0}, v_{5}\right),\left(v_{0}, v_{6}\right),\left(v_{1}, v_{4}\right),\left(v_{1}, v_{6}\right),\left(v_{2}, v_{4}\right),\left(v_{2}, v_{5}\right),\left(v_{3}, v_{5}\right),\left(v_{3}, v_{6}\right)\right\}$. All edges are of unit length, see Figure 2.

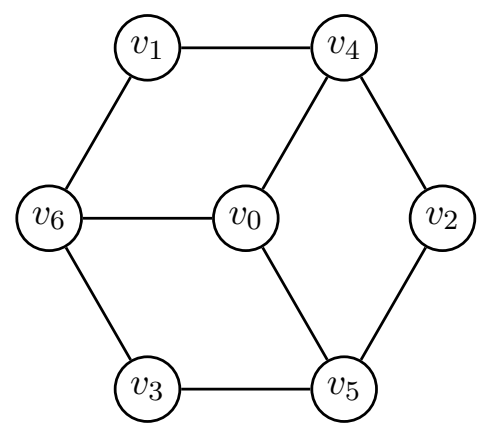

Figure 2: Graph in Example 2.3.

Set $X=A(G)$. Consider the game $\left(N, v_{I I}\right)$, defined on $X$, with $N_{0}=\left\{v_{0}, v_{1}, v_{2}, v_{3}\right\}$ and $N=\left\{v_{1}, v_{2}, v_{3}\right\}$. It is easy to check that for each coalition $S \subseteq N$ with $|S| \leq 2$ we have $v_{I I}(S)=2$, and $v_{I I}(N)=4$.

By symmetry, if the core was not empty the symmetric allocation $x=(4 / 3,4 / 3,4 / 3)$ would be in the core contradicting the constraint $x_{1}+x_{2} \leq v_{I I}\left(\left\{v_{1}, v_{2}\right\}\right)=2$.

For any metric space $X$, the definition of $v_{I I}$ ensures the monotonicity of the game $\left(N, v_{I I}\right)$, whereas subadditivity is proved in the next proposition, under the following continuity assumption:

Definition 2.1 Let $X$ be a metric space such that for any pair of points $x, y \in X$, and a real $0 \leq \alpha \leq 1$, there is a point $z \in X$ such that $d(x, z)+d(z, y)=d(x, y)$ and $d(x, z)=\alpha d(x, y)$. Then $X$ is called a "geodesic metric space", Papadopoulos (2005). 
Proposition 2.1 If $X$ is a geodesic metric space, then the radius game $\left(N, v_{I I}\right)$ over $X$ is subadditive.

Proof. Consider a pair of coalitions, $S_{1}$ and $S_{2}$. We need to show that

$$
v_{I I}\left(S_{1} \cup S_{2}\right) \leq v_{I I}\left(S_{1}\right)+v_{I I}\left(S_{2}\right) .
$$

For $j=1,2$, let $c_{j}$ and $r_{j}$ be the 1-center and 1-radius of the smallest ball enclosing the points in $S_{j} \cup\left\{v_{0}\right\}$, respectively.

Let $P\left(c_{1}, c_{2}\right)$ be a shortest path in $X$, connecting $c_{1}$ and $c_{2}$. Let $d\left(c_{1}, c_{2}\right)$ denote the length of $P\left(c_{1}, c_{2}\right)$. Then, $d\left(c_{1}, c_{2}\right) \leq d\left(c_{1}, v_{0}\right)+d\left(v_{0}, c_{2}\right) \leq r_{1}+r_{2}$.

Suppose without loss of generality that $r_{2} \geq r_{1}$. If $r_{2} \geq r_{1}+d\left(c_{1}, c_{2}\right)$, then a center established at $c_{2}$ will ensure a covering radius of $r_{2}$ to all nodes in $S_{1} \cup S_{2} \cup\left\{v_{0}\right\}$. Hence, $v_{I I}\left(S_{1} \cup S_{2}\right) \leq 2 r_{2}=v_{I I}\left(S_{2}\right)$.

If $r_{1} \leq r_{2} \leq r_{1}+d\left(c_{1}, c_{2}\right)$, then consider a center established at the point $c^{*}$, such that $d\left(c_{1}, c^{*}\right)=\left(d\left(c_{1}, c_{2}\right)+r_{2}-r_{1}\right) / 2$, and $d\left(c_{2}, c^{*}\right)=\left(d\left(c_{1}, c_{2}\right)-r_{2}+r_{1}\right) / 2$. It is easy to check that this center will ensure a covering radius of $\left(d\left(c_{1}, c_{2}\right)+r_{1}+r_{2}\right) / 2 \leq r_{1}+r_{2}$ to all nodes in $S_{1} \cup S_{2} \cup\left\{v_{0}\right\}$. (Note that $v_{0}$ is in the intersection of the smallest balls enclosing $S_{1} \cup\left\{v_{0}\right\}$ and $S_{2} \cup\left\{v_{0}\right\}$.) Therefore, $v_{I I}\left(S_{1} \cup S_{2}\right) \leq v_{I I}\left(S_{1}\right)+v_{I I}\left(S_{2}\right)$.

\section{$2.1 \quad 1 / 2$-budget balanced allocations}

As illustrated in previous examples, the core of the radius game can be empty even for subadditive games. To address games with empty core, various cost shares have been defined. One of them is the concept of $\gamma$-budget balanced cost allocation defined in Caprara and Letchford (2010). Given a real $\gamma$, a vector $x$ is a $\gamma$-budget balanced allocation of the radius game $\left(N, v_{I I}\right)$ if

$$
\sum_{j: v_{j} \in S} x_{j} \leq v_{I I}(S), \forall S \subseteq N
$$

and

$$
\sum_{j: v_{j} \in N} x_{j} \geq \gamma v_{I I}(N) .
$$

It is noted in Caprara and Letchford (2010) that recently, researchers have devoted some attention to the problem of finding an allocation which is $\gamma$-budget balanced for the maximum possible $\gamma$. This problem is called the optimal cost share problem (OCSP).

The relationship between the MRLG and the MDLG implies that every vector in $C\left(N, v_{I}\right)$ is also a $1 / 2$-budget balanced allocation of the radius game $\left(N, v_{I I}\right)$. Specifically, the inequal- 
ity $v_{I}(S) \leq v_{I I}(S) \leq 2 v_{I}(S)$ (based on (1)) implies that if $x \in C\left(N, v_{I}\right)$ then, for any $S \subseteq N$,

$$
\sum_{j: v_{j} \in S} x_{j} \leq v_{I}(S) \leq v_{I I}(S)
$$

and

$$
\sum_{j: v_{j} \in N} x_{j}=v_{I}(N) \geq(1 / 2) v_{I I}(N)
$$

Another 1/2-budget balanced allocation, which may not be in $C\left(N, v_{I}\right)$, can be obtained as follows:

In general, $v_{I I}(N)$ is bounded below by $D\left(N_{0}\right)$, and therefore also by the maximum distance from $v_{0}$ to the points of $N$. It is bounded above by the sum of the two largest entries in $\left\{d\left(v_{i}, v_{0}\right)\right\}, v_{i} \in N$. Hence,

$$
\max _{v_{i} \in N} d\left(v_{i}, v_{0}\right) \leq v_{I I}(N) \leq 2 \max _{v_{i} \in N} d\left(v_{i}, v_{0}\right) .
$$

Suppose that $d\left(v_{q}, v_{0}\right)=\max _{v_{i} \in N} d\left(v_{i}, v_{0}\right)$. Then, clearly, the allocation $x$ defined by $x_{q}=$ $d\left(v_{q}, v_{0}\right)$ and $x_{i}=0$, for any $v_{i} \in N, i \neq q$, is $1 / 2$-budget balanced.

Given an arbitrary radius game with an empty core, the value $\gamma=1 / 2$ is not always an optimal solution to the respective OCSP. (See examples 2.1, 2.2 and 2.3 , where the optimal value for OCSP is $\gamma=3 / 4$.) Nevertheless, the results in Sections 3-5 about the polynomial representation of the core imply that if a radius game is defined on a discrete metric space, the $\ell_{p}$ metric space defined over $\mathbb{R}^{\mathrm{d}}$, or the network metric space $A(G)$, the solution to OCSP can be found in polynomial time by solving a single linear program with $|N|$ variables and a polynomial number of constraints.

\section{Network metric spaces}

We now consider some specific metric spaces that are frequently studied in location analysis and show that in these cases the core can be represented by a polynomial number of linear inequalities. Note that in general we need an exponential number of linear inequalities to represent the core of a game, (2).

Consider first the case where $X=A(G)$, the metric space induced by an undirected connected graph $G=(V, E), V=\left\{v_{0}, v_{1}, \ldots, v_{n}\right\}$, and its positive edge lengths.

Assume that the set of players $N$ satisfies $N \subseteq V \backslash\left\{v_{0}\right\}$. Moreover, to be consistent with the notation introduced above, suppose without loss of generality, that $N=\left\{v_{1}, v_{2}, \ldots, v_{k}\right\}$, where $k=|N|$.

We will show that in this case, there is an efficient representation of the core of the radius game $\left(N, v_{I I}\right)$, involving $O\left(m|N|^{2}\right)$ constraints, where $m=|E|$. Such a representation implies 
that membership in the core, as well as its nonemptiness can be tested in strongly polynomial time by the algorithm in Tardos (1986).

Proposition 3.1 Consider the radius game $\left(N, v_{I I}\right)$, defined on a network metric space $A(G)$, induced by a graph $G=(V, E)$ and its positive edge lengths. Then, there is a collection of subsets of $N,\left\{S_{p, q}^{i, j}\right\},\left(v_{i}, v_{j}\right) \in E, v_{p}, v_{q} \in N \cup\left\{v_{0}\right\},(i, j, p, q) \in I$, such that $|I|=O\left(m|N|^{2}\right)$ and

$$
C\left(N, v_{I I}\right)=\left\{x \in \mathbb{R}_{+}^{N}: x(N)=v_{I I}(N), x\left(S_{p, q}^{i, j}\right) \leq v_{I I}\left(S_{p, q}^{i, j}\right), \forall(i, j, p, q) \in I\right\} .
$$

As a result, membership in the core, as well as nonemptiness of the core, can be tested in strongly polynomial time.

Proof. First we note that, if $N=\left\{v_{1}, \ldots, v_{n}\right\}, v_{I I}(N)$ is equal to the diameter of a minimum diameter spanning tree of $V$, (Handler (1973) and Hassin and Tamir (1995)). This spanning tree, say $T^{*}$, solves the continuous (or absolute) 1 -center problem on $G$, and it can be found in $O\left(m n+n^{2} \log n\right)$ time.

More generally, when $N \subseteq V \backslash\left\{v_{0}\right\}, v_{I I}(S)$ is defined as the diameter length of a minimum diameter spanning tree of $S \cup\left\{v_{0}\right\}, \forall S \subseteq N$. Such a tree, say $T^{*}(S)$, solves the continuous 1-center problem for the subset of nodes $S \cup\left\{v_{0}\right\}$, and it can be found in $O\left(m n+n^{2} \log n\right)$ time, (Hassin and Tamir (1995)). Recall that the continuous 1-center problem for some subset $V^{\prime} \subseteq V$, defines the smallest radius neighborhood in the metric space $A(G)$, which covers $V^{\prime}$.

Moreover, $T^{*}(S)$ has the following property. There is an edge of $G$, say $\left(v_{i}, v_{j}\right)$, such that the 1-center of $T^{*}(S)$ is on this edge, see Hassin and Tamir (1995), and

$$
v_{I I}(S)=d\left(v_{p}, v_{i}\right)+l\left(v_{i}, v_{j}\right)+d\left(v_{j}, v_{q}\right),
$$

for some nodes $v_{p}, v_{q} \in S \cup\left\{v_{0}\right\}$.

In total there are at most $O\left(|N|^{2}\right)$ centers of minimum diameter trees on the edge $\left(v_{i}, v_{j}\right)$. Each such center $c_{p, q}^{i, j}$ is associated with a radius of the form

$$
r_{p, q}^{i, j}=\left(d\left(v_{p}, v_{i}\right)+l\left(v_{i}, v_{j}\right)+d\left(v_{j}, v_{q}\right)\right) / 2 .
$$

Next, for each center $c_{p, q}^{i, j}$ such that $d\left(v_{0}, c_{p, q}^{i, j}\right) \leq r_{p, q}^{i, j}$, define the maximal coalition

$$
S_{p, q}^{i, j}=\left\{u \in N: d\left(u, c_{p, q}^{i, j}\right) \leq r_{p, q}^{i, j}\right\}
$$

Let

$$
I=\left\{(i, j, p, q):\left(v_{i}, v_{j}\right) \in E, v_{p}, v_{q} \in N \cup\left\{v_{0}\right\}, d\left(v_{0}, c_{p, q}^{i, j}\right) \leq r_{p, q}^{i, j}\right\}
$$


Next consider a coalition $S \subseteq N$. Then, from the above discussion it follows that there is some center $c_{p, q}^{i, j}$ and radius $r_{p, q}^{i, j}$, such that $v_{I I}(S)=2 r_{p, q}^{i, j}$, and $S \subseteq S_{p, q}^{i, j}$. Hence, the monotonicity of the game implies that the core constraint $x(S) \leq v_{I I}(S)$ is implied by the constraint $x\left(S_{p, q}^{i, j}\right) \leq v_{I I}\left(S_{p, q}^{i, j}\right)$. This validates the efficient representation of the core stated in the proposition. Finally, note that using the above algorithms for solving 1-center problems, it takes polynomial time to construct the entire collection $\left\{S_{p, q}^{i, j}\right\},\left(v_{i}, v_{j}\right) \in E, v_{p}, v_{q} \in N \cup\left\{v_{0}\right\}$, $(i, j, p, q) \in I$. In particular, membership in the core, as well as its nonemptiness can be tested in strongly polynomial time by the algorithm in Tardos (1986). This completes the proof.

Remark 3.1 Dealing with MRLG defined on a network metric space we can assume without loss of generality that the underlying graph $G=(V, E)$ is a complete graph, and its edge lengths satisfy the triangle inequality. Otherwise, we can always introduce an edge between any pair of nodes and set its length equal to the distance in $A(G)$ between the pair. Consider a coalition $S$. We note that in this case each simple path of $T^{*}(S)$ has at most 3 edges. However, even in this case $T^{*}(S)$ is not necessarily a subtree of $G_{v_{0}}(S)$, the subgraph of $G$ induced by the node set $S \cup\left\{v_{0}\right\}$. As an example consider the complete graph with node set $V=\left\{v_{0}, v_{1}, v_{2}, v_{3}\right\}$. Let the length of the edges $\left(v_{3}, v_{0}\right),\left(v_{3}, v_{1}\right),\left(v_{3}, v_{2}\right)$ be equal to 1 , and the length of the other 3 edges equal to 2 . When $S=\left\{v_{1}, v_{2}\right\}, T^{*}(S)$ is the star centered at $v_{3}$, which is not in $G_{v_{0}}(S)$.

Corollary 3.1 If $G$ is a tree, there is a collection of subsets of $N,\left\{S_{p, q}\right\}, v_{p}, v_{q} \in N \cup\left\{v_{0}\right\}$, $p, q \in I^{\prime}$, such that $\left|I^{\prime}\right|=O\left(|N|^{2}\right)$, and the core of the game $\left(N, v_{I I}\right)$ is defined by

$$
C\left(N, v_{I I}\right)=\left\{x \in \mathbb{R}_{+}^{N}: x(N)=v_{I I}(N), x\left(S_{p, q}\right) \leq v_{I I}\left(S_{p, q}\right) \forall p, q \in I^{\prime}\right\}
$$

Proof. If $G$ is a tree the total number of centers of relevant minimum diameter spanning subtrees is only $O\left(|N|^{2}\right)$. In this case each pair of nodes, $v_{p}, v_{q}$ contributes one candidate center, denoted by $c_{p, q}$, the midpoint of the unique simple path connecting $v_{p}$ with $v_{q}$. If $d\left(v_{0}, c_{p, q}\right) \leq d\left(v_{p}, v_{q}\right) / 2$, the respective maximal coalition is then defined by

$$
S_{p, q}=\left\{u \in N: d\left(u, c_{p, q}\right) \leq d\left(v_{p}, v_{q}\right) / 2\right\}
$$

Set $I^{\prime}=\left\{(p, q): d\left(v_{0}, c_{p, q}\right) \leq d\left(v_{p}, v_{q}\right) / 2\right\}$. Then, the general result in Proposition 3.1 leads to the simpler description of $C\left(N, v_{I I}\right)$ stated in the corollary.

Also, when $G$ is a tree the radius and the diameter games coincide, i.e., $v_{I}(S)=v_{I I}(S)$ for any $S \subseteq N$. As shown in our companion paper, Puerto et al. (2010), the radius game is submodular in this case, its core is always nonempty, and both its Shapley value and nucleolus can be computed in polynomial time. 
Remark 3.2 Given an undirected connected graph $G=(V, E)$, with $V=\left\{v_{0}, v_{1}, \ldots, v_{n}\right\}$, we observe that the respective metric space $X=A(G)$, induced by $G$ and its positive edge lengths, is geodesic. Example 2.3 illustrates that when the set of players $N$ is a proper subset of $V \backslash\left\{v_{0}\right\}=\left\{v_{1}, \ldots, v_{n}\right\}$, the core of the radius location game can be empty. However, we still do not know whether the core of the game $\left(N, v_{I I}\right)$ defined on the space $X=A(G)$ is always nonempty in the case where $G=(V, E)$ is a general connected undirected graph and $N=\left\{v_{1}, \ldots, v_{n}\right\}$. We will refer to this case as the Complete Minimum Radius Location game (CMRLG) on networks.

We now focus on some observations and special cases of the complete radius game, CMRLG. First we note that the function $v_{I I}$ may not be submodular if the graph $G$ contains a cycle.

Example 3.1 Consider a 4-cycle with unit edge lengths, and $V=\left\{v_{0}, v_{1}, v_{2}, v_{3}\right\}$, see Figure 3 .

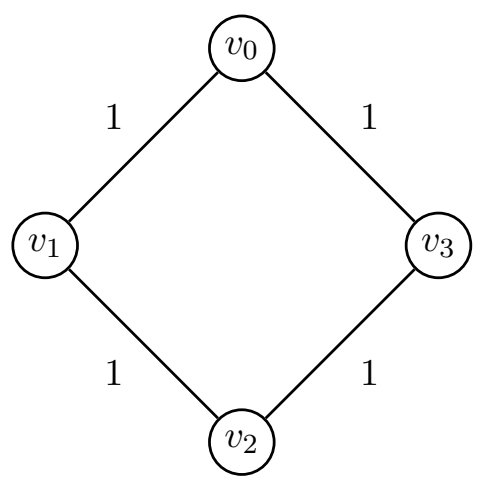

Figure 3: Graph in Example 3.1

Let $S_{1}=\left\{v_{1}, v_{2}\right\}$ and $S_{2}=\left\{v_{3}, v_{2}\right\}$. Then, $v_{I I}\left(S_{1}\right)=v_{I I}\left(S_{2}\right)=2, v_{I I}\left(S_{1} \cup S_{2}\right)=3$, $v_{I I}\left(S_{1} \cap S_{2}\right)=2$, and therefore $v_{I I}\left(S_{1} \cup S_{2}\right)+v_{I I}\left(S_{1} \cap S_{2}\right)>v_{I I}\left(S_{1}\right)+v_{I I}\left(S_{2}\right)$.

We have noted in the Introduction that there is a core allocation for the diameter game $\left(N, v_{I}\right)$ splitting $v_{I}(N)$ between a pair of players corresponding to the diameter of $V$. In contrast, the core of the complete radius location game $\left(N, v_{I I}\right)$ may not in general contain an allocation where only 2 players pay a positive cost. For instance, in Example $3.1 d\left(v_{0}, v_{2}\right)=2$, and $d\left(v_{0}, v_{1}\right)=d\left(v_{0}, v_{3}\right)=1$. However the unique core allocation is the vector $(1,1,1)$.

We also note in passing that in our attempts to prove that the core of the CMRLG is nonempty, we have produced more involved examples, indicating that in general there is no fixed number $c$, such that for any $n$, there is a core allocation, where the number of players paying a positive cost is bounded by $c$.

To summarize, we still do not know whether the core of the MRLG is always nonempty for the case where $X=A(G)$ and $N=V \backslash\left\{v_{0}\right\}$. 
We have proven the nonemptiness of the core only for the case where $G=K_{4}$, i.e., when there are 3 players. (Our proof, based on a tedious case analysis, can be obtained from the authors upon request.)

Next we consider two families of graphs for which the respective radius games have nonempty cores. We use the following observation.

Remark 3.3 Given the metric space $X$ and the set of points $N_{0}$, consider the games $\left(N, v_{I}\right)$ and $\left(N, v_{I I}\right)$. For each subset $S \subseteq N, v_{I}(S) \leq v_{I I}(S)$. Therefore, if $v_{I}(N)=v_{I I}(N)$, $C\left(N, v_{I}\right) \subseteq C\left(N, v_{I I}\right)$. For example, for any graph $G=(V, E)$, if $X=A(G)$ and $V$ satisfies $D(V)=2 R(V)$, then $C\left(N, v_{I I}\right)$ is nonempty. This holds for the class of median graphs (which includes all tree graphs), and the class of graphs with "long diameters".

\subsection{Median graphs}

A median graph $G=(V, E)$ is defined by the following property, (Mulder $(1978,1980)$ ). For any triplet of nodes, $\{x, y, z\}$, there is a unique node, $v=m(x, y, z)$, called the median of $\{x, y, z\}$, which is in the intersection of the node sets of all shortest paths connecting distinct pairs of nodes from the triplet $\{x, y, z\}$.

Lemma 3.1 Let $G=(V, E)$ be a median graph with positive edge lengths. Then, $v_{I}(N)=$ $D(V)=2 R(V)=v_{I I}(N)$ in the metric space $X=A(G)$.

Proof. To prove that for a median graph $G=(V, E), D(V)=2 R(V)$, consider $P(x, y)$ to be a longest path in a minimum diameter spanning tree $T^{*}$ of $G$. From the triangle inequality $D(V) \leq d_{T^{*}}(x, y)=2 R(V)$. It is therefore sufficient to show that $d_{G}(x, y)$, the distance from $x$ to $y$ in $A(G)$ is equal to $2 R(V)$.

Let $z$ be the 1-center defined as the middle point of $P(x, y)$, (Hassin and Tamir (1995)). Suppose without loss of generality that $z$ is a node. (Otherwise, augment it to the node set of $G$. The new graph is still median, and its diameter is equal to that of $G$.)

Now apply the median property to the triplet $\{x, y, z\}$. From the definition of $z$ as a center, the two subpaths of $P(x, y)$ in $T^{*}$, connecting $z$ with $x$, and $z$ with $y$ are in fact, respectively, shortest paths in $G$, between $z$ and $x$, and between $z$ and $y$. Since $z$ is the only node which is in both subpaths, $z$ must be the median of $\{x, y, z\}$. Hence, $z$ must be on every shortest path in $G$, connecting $x$ and $y$. Therefore, $d_{G}(x, z)+d_{G}(z, y)=d_{G}(x, y)$. But the right hand side of the latter equation is, by definition, equal to $2 R(V)$.

\subsection{Long-diameter graphs}

Let $G=(V, E)$ be an undirected and connected graph with positive edge lengths $\left\{l_{e}\right\}, e \in E$. For each node $v \in V$ define $f(v)$ to be the maximum of the lengths of all edges which are 
incident to $v$. $G$ is called a long-diameter graph if $D(V) \geq(2 / 3) \sum_{v \in V} f(v)$. For example, if all edges are of unit length, $G$ is such a graph if $D(V) \geq 2|V| / 3$. It has been recently proved by Liu and Huang (2009), that for a long-diameter graph $G=(V, E), D(V)=2 R(V)$. Hence, from the above discussion we can conclude that for long-diameter graphs, the core of the minimum radius location game is nonempty.

\subsection{MSTG and MRLG}

In the case of a network metric space it is interesting to compare the MRLG with the related minimum length spanning tree game (MSTG), Megiddo (1978) and Granot and Huberman $(1981,1984)$. As we noted above, in the $\operatorname{MRLG}\left(N, v_{I I}\right)$ the characteristic function is defined by the minimum diameter spanning Steiner subtree while in the MSTG, which we denote by $\left(N, v^{\prime}\right)$, the characteristic function $v^{\prime}(S)$ is defined by the minimum length Steiner subtree spanning $S \cup\left\{v_{0}\right\}$. By definition, we have $v_{I I}(S) \leq v^{\prime}(S)$ for any coalition $S$. Therefore, if $v_{I I}(N)=v^{\prime}(N)$, the core of the MRLG is contained in the core of the minimum length spanning tree game. Specifically, the latter holds if the minimum diameter spanning tree of $V$ is a spanning path.

In general, it is known that when $N$ is a proper subset of $V \backslash\left\{v_{0}\right\}, C\left(N, v^{\prime}\right)$, the core of the MSTG can be empty, see Tamir (1991). Moreover, testing membership in $C\left(N, v^{\prime}\right)$ is NP-hard, see Faigle et al., (1997).

Consider the complete case, i.e., $N=V \backslash\left\{v_{0}\right\}$. Then, given some minimum length spanning tree $T^{* *}$ of $N \cup v_{0}$, there is a natural allocation in $C\left(N, v^{\prime}\right)$ that does not depend on the edges outside $T^{* *}$. In this core allocation, the cost allocated to node $v_{i}$ is the length of the unique edge incident to $v_{i}$ which is on the (unique) path of $T^{* *}$, connecting $v_{i}$ to $v_{0}$, Bird (1976), Granot and Huberman (1981, 1984).

This is not the case for the MRLG. All core allocations may depend on edges outside $T^{*}$, as shown in the following example.

Example 3.2 Consider the graph $G=(V, E)$ where

$$
V=\left\{v_{0}, v_{1}, v_{2}, v_{3}\right\}, E=\left\{\left(v_{0}, v_{1}\right),\left(v_{0}, v_{3}\right),\left(v_{1}, v_{2}\right),\left(v_{1}, v_{3}\right),\left(v_{2}, v_{3}\right)\right\}
$$

The edge lengths are $l\left(v_{0}, v_{1}\right)=0.5, l\left(v_{0}, v_{3}\right)=l\left(v_{2}, v_{3}\right)=4, l\left(v_{1}, v_{2}\right)=7, l\left(v_{1}, v_{3}\right)=4+\epsilon$, see Figure 4.

The unique minimum diameter spanning tree of $G, T^{*}$, is defined by the star centered at $v_{3}$. The diameter of $G$ is attained by the pair of nodes $v_{0}$ and $v_{2}$. Any core allocation cannot charge $v_{1}$ more than 0.5 . Hence, if a core allocation ignores the edges outside $T^{*}$, and assigns charges which are distances on $T^{*}, v_{1}$ will have to be charged 0 . But then $v_{2}$ and $v_{3}$ together cannot be charged more than 8 , which is less than $D\left(T^{*}\right)=8+\epsilon$. 


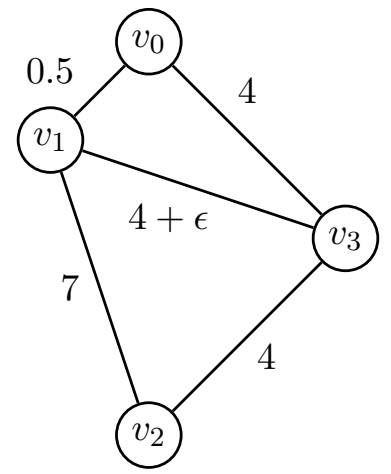

Figure 4: Graph in Example 3.2.

One extreme allocation in the core is defined by the vector $\left(x_{1}, x_{2}, x_{3}\right)=(\epsilon, 4,4)$. The other three core extreme points are $(\epsilon, 7.5-\epsilon, 0.5+\epsilon),(0.5,7, .5+\epsilon),(0.5,3.5-\epsilon, 4)$.

\section{Discrete metric spaces}

We have already noted above that when the underlying metric space $X$ consists of a finite number of points, the respective MRLG may not even be subadditive and players may not have incentives to cooperate, e.g., the core $C\left(N, v_{I I}\right)$ can be empty. Nevertheless, in the discrete finite case $C\left(N, v_{I I}\right)$ has a compact representation, as shown in the next proposition, and therefore its nonemptiness can be tested efficiently.

Proposition 4.1 Suppose that $X=\left\{v_{0}, v_{1}, \ldots, v_{n}\right\}$ is a finite metric space, and let $N \subseteq$ $X \backslash\left\{v_{0}\right\}$. For each $i=0,1, \ldots, n$, and $v_{p} \in N \cup\left\{v_{0}\right\}$, define $r_{p}^{i}=d\left(v_{i}, v_{p}\right)$. If $d\left(v_{0}, v_{i}\right) \leq r_{p}^{i}$, define

$$
S_{p}^{i}=\left\{u \in N: d\left(u, v_{i}\right) \leq r_{p}^{i}\right\} .
$$

Let $I=\left\{(i, p): d\left(v_{0}, v_{i}\right) \leq r_{p}^{i}\right\}$. Then,

$$
C\left(N, v_{I I}\right)=\left\{x \in R_{+}^{N}: x(N)=v_{I I}(N), x\left(S_{p}^{i}\right) \leq v_{I I}(S), \forall(i, p) \in I\right\} .
$$

Proof. Consider a coalition $S \subseteq N$. Then, there is some $v_{i} \in X$, and $v_{p} \in N \cup\left\{v_{0}\right\}$, such that $v_{I I}(S)=2 d\left(v_{i}, v_{p}\right)=2 r_{p}^{i}$. By definition $S \subseteq S_{p}^{i}$. Using the monotonicity of the game, we conclude that the core constraint $x(S) \leq v_{I I}(S)$ is dominated by the constraint $x\left(S_{p}^{i}\right) \leq v_{I I}\left(S_{p}^{i}\right)$. This completes the proof. 


\section{$5 \quad \ell_{p}$ metric spaces over $\mathbb{R}^{\mathrm{d}}$}

In this section we focus on the case in which the MRLG $\left(N, v_{I I}\right)$ is defined on the $\ell_{p}$ metric space over $\mathbb{R}^{\mathrm{d}}$. Again, we let $N_{0}=V=\left\{v_{0}, v_{1}, \ldots, v_{n}\right\}$ be a set of points in $\mathbb{R}^{\mathrm{d}}$, and set $N=V \backslash\left\{v_{0}\right\}$.

The following examples show that in general the MRLG is not submodular, and that with the exception of the case $p=\infty, v_{I}(N)=D(V) \neq 2 R(V)=v_{I I}(N)$. Hence, the existence of core allocations is not clear in the case where $p \neq \infty$.

Example 5.1 Consider the planar $\ell_{p}$ normed case with $V=\left\{v_{0}, v_{1}, v_{2}, v_{3}\right\}$, where, $v_{0}=$ $(0,0), v_{1}=(0,1), v_{2}=(1,0)$ and $v_{3}=(-1,0)$.

We have $v_{I I}\left(\left\{v_{1}, v_{2}, v_{3}\right\}\right)=2, v_{I I}\left(\left\{v_{1}\right\}\right)=1$, and $v_{I I}\left(\left\{v_{1}, v_{2}\right\}\right)=v_{I I}\left(\left\{v_{1}, v_{3}\right\}\right)=2^{1 / p}$. Thus, $v_{I I}$ is not submodular in this example for any $p$ such that $2^{1 / p}<3 / 2$, which in particular applies to $2 \leq p \leq \infty$.

Example 5.2 Consider the planar $\ell_{1}$ case with $V=\left\{v_{0}, v_{1}, v_{2}, v_{3}\right\}$, where, $v_{0}=(0,0)$, $v_{1}=(1,-1), v_{2}=(1,1)$ and $v_{3}=(-1,-1)$. We have $v_{I I}\left(\left\{v_{1}, v_{2}, v_{3}\right\}\right)=4, v_{I I}\left(\left\{v_{1}\right\}\right)=2$, and $v_{I I}\left(\left\{v_{1}, v_{2}\right\}\right)=v_{I I}\left(\left\{v_{1}, v_{3}\right\}\right)=2$. Thus, $v_{I I}$ is not submodular in this case.

The next two examples show that for any $1<p<\infty$ in the planar case, and for the rectilinear norm $\ell_{1}$, even in $\mathbb{R}^{3}, v_{I I}(N)=2 R\left(N \cup\left\{v_{0}\right\}\right)$ can be strictly larger than $v_{I}(N)=$ $D\left(N \cup\left\{v_{0}\right\}\right)$. (In $\mathbb{R}^{2}$ the $\ell_{1}$ norm is equivalent to the $\ell_{\infty}$ norm.)

Example 5.3 Consider the set of points $V=\left\{v_{0}, v_{1}, v_{2}, v_{3}\right\}$ where $v_{1}=(a, b), v_{2}=(-a, b)$, $v_{3}=(0,-1)$, and $v_{0}=(0,0)$. For $1<p<\infty$, let $a=b=2^{-1 / p}$. Then, the $\ell_{p}$ diameter of $V$ is $\left(a^{p}+(b+1)^{p}\right)^{1 / p}$ whereas the $\ell_{1}$ radius is 1 and the 1 -center is $(0,0)$. Hence, $v_{I}(N)=D(V)<b+1<2=2 R(V)=v_{I I}(N)$.

Example 5.4 Consider the set of points $V=\left\{v_{0}, v_{1}, v_{2}, v_{3}\right\}$ where $v_{1}=(1,1,1), v_{2}=$ $(-1,-1,1), v_{3}=(-1,1,-1)$, and $v_{0}=(1,-1,-1)$. The $\ell_{1}$ diameter of $V$ is 4 whereas the $\ell_{1}$ radius is 3 and the 1 -center is $(0,0,0)$. Hence, $v_{I}(N)=D(V)<2 R(V)=v_{I I}(N)$.

We first show that for any $p \geq 1$, the core of the game $\left(N, v_{I I}\right)$, defined on the $\ell_{p}$ metric space over $\mathbb{R}^{\mathrm{d}}$, can be represented as a set described by a polynomial number of linear inequalities, for any fixed $d$.

Consider first the case where $1<p<\infty$.

Theorem 5.1 Let $1<p<\infty$, and consider the game $\left(N, v_{I I}\right)$, defined on the $\ell_{p}$ metric space over $\mathbb{R}^{\mathrm{d}}$. Let $\left\{S_{j}\right\}, j \in J$, be the collection of all subsets $S \subseteq N$ with $|S| \leq \mathrm{d}+1$. For 
each $j \in J$, let $B\left(S_{j}\right)$, be the smallest enclosing ball containing $S_{j} \cup\left\{v_{0}\right\}$, and let $S_{j}^{\prime}$ be the subset of all points in $N$, contained in $B\left(S_{j}\right)$. Then the core of the game is given by,

$$
C\left(N, v_{I I}\right)=\left\{x \in \mathbb{R}_{+}^{n}: x\left(S_{j}^{\prime}\right) \leq v_{I I}\left(S_{j}\right), \forall j \in J, \text { and } x(N)=v_{I I}(N)\right\}
$$

Proof. For any subset $S \subseteq N, v_{I I}(S)$ is the diameter of $B(S)$, a smallest enclosing ball containing $S \cup\left\{v_{0}\right\}$. (Since $1<p<\infty, B(S)$ is unique, Zurcher (2007).)

By the Helly property there is a subset $S_{j} \subseteq S, j \in J$, such that $v_{I I}(S)=v_{I I}\left(S_{j}\right)$. Then, by definition $S \subseteq S_{j}^{\prime}$. Moreover, by the monotonicity of the game each vector in the core is nonnegative, and therefore $x(S) \leq x\left(S_{j}^{\prime}\right)$. Hence, the constraint $x(S) \leq v_{I I}(S)$ is dominated by the constraint $x\left(S_{j}^{\prime}\right) \leq v_{I I}\left(S_{j}\right)$. This completes the proof.

Next, consider the case where $p=\infty$. As above, let $\left\{S_{j}\right\}, j \in J$, be the collection of all subsets $S \subseteq N$ with $|S| \leq \mathrm{d}+1$.

Theorem 5.2 Consider the game $\left(N, v_{I I}\right)$, defined on the $\ell_{\infty}$ metric space over $\mathbb{R}^{\mathrm{d}}$. Then there is a collection of subsets of $N,\left\{S_{j}^{\infty}(k)\right\}, j \in J, k=1, \ldots, c_{j}^{\infty}(n, \mathrm{~d})$, such that $c_{j}^{\infty}(n, \mathrm{~d})=$ $O\left(2^{\mathrm{d}} n^{(\mathrm{d}-1)}\right)$, and the core of the game is given by,

$C\left(N, v_{I I}\right)=\left\{x \in \mathbb{R}_{+}^{n}: x\left(S_{j}^{\infty}(k)\right) \leq v_{I I}\left(S_{j}\right), \forall j \in J, k=1, \ldots, c_{j}^{\infty}(n, \mathrm{~d})\right.$ and $\left.x(N)=v_{I I}(N)\right\}$.

Proof. For each subset $S$ the problem of finding the smallest $\ell_{\infty}$ ball enclosing $S$ is reduced to finding a smallest hypercube containing $S$. Such a hypercube is not unique. The set of centers of all optimal hypercubes is itself a hypercube of dimension less than or equal to $\mathrm{d}-1$. For $j \in J$ consider an optimal hypercube $H\left(S_{j}\right)$ enclosing $S_{j} \cup\left\{v_{0}\right\}$ and let $P\left(H\left(S_{j}\right)\right)$ be the maximal subset of $N$, contained in $H\left(S_{j}\right)$. We can shift $H\left(S_{j}\right)$ along the axes and obtain an optimal hypercube $H^{\prime}\left(S_{j}\right)$ such that $P\left(H^{\prime}\left(S_{j}\right)\right)=P\left(H\left(S_{j}\right)\right)$, and for each coordinate $i=1, \ldots, d$, one of the two faces of $H^{\prime}\left(S_{j}\right)$ corresponding to the $i^{\text {th }}$ coordinate contains a point in $N$. Thus, there is only $c_{j}^{\infty}(n, \mathrm{~d})=O\left(2^{\mathrm{d}} n^{(\mathrm{d}-1)}\right)$ such maximal subsets of $N$, associated with a given subset $S_{j}, j \in J$. Denote this collection of subsets by $\left\{S_{j}^{\infty}(k)\right\}, k=1, \ldots, c_{j}^{\infty}(n, \mathrm{~d})$.

Using the monotonicity of the game and following the arguments used in the previous proof, we observe that for each subset $S \subseteq N$, there is a subset $S_{j}, j \in J$, and $k=1, \ldots, c_{j}^{\infty}(n, \mathrm{~d})$, such that the constraint $x(S) \leq v_{I I}(S)$, is dominated by the constraint $x\left(S_{j}^{\infty}(k)\right) \leq v_{I I}\left(S_{j}\right)$. This completes the proof.

A similar analysis applies to the rectilinear case when $p=1$.

Theorem 5.3 Consider the game $\left(N, v_{I I}\right)$, defined on the $\ell_{1}$ metric space over $\mathbb{R}^{\mathrm{d}}$. Then there is a collection of subsets of $N,\left\{S_{j}^{1}(k)\right\}, j \in J, k=1, \ldots, c_{j}^{1}(n, \mathrm{~d})$, such that $c_{j}^{1}(n, \mathrm{~d})=$ $O\left(2^{\mathrm{d}^{2}} n^{\mathrm{d}-1}\right)$, and the core of the game is given by,

$$
C\left(N, v_{I I}\right)=\left\{x \in \mathbb{R}_{+}^{n}: x\left(S_{j}^{1}(k)\right) \leq v_{I I}\left(S_{j}\right), \forall j \in J, k=1, \ldots, c_{j}^{1}(n, \mathrm{~d}) \text { and } x(N)=v_{I I}(N)\right\} .
$$


Proof. The proof goes along the lines of the previous proof and is therefore outlined only. In this case an $\ell_{1}$ enclosing ball is a polyhedron with $2^{\mathrm{d}}$ faces. Again, by shifting an enclosing ball of a subset $S_{j}, j \in J$, along the normals to the facets, each given subset $S_{j}, j \in J$, is associated with $c_{j}^{1}(n, \mathrm{~d})$ maximal subsets of $N$, where $c_{j}^{1}(n, \mathrm{~d})=O\left(2^{\mathrm{d}^{2}} n^{\mathrm{d}-1}\right)$. Denote this collection of subsets by $\left\{S_{j}^{1}(k)\right\}, k=1, \ldots, c_{j}^{1}(n, \mathrm{~d})$.

As above, we observe that for each subset $S \subseteq N$, there is a subset $S_{j}, j \in J$, and $k=1, \ldots, c_{j}^{1}(n, \mathrm{~d})$, such that the constraint $x(S) \leq v_{I I}(S)$, is dominated by the constraint $x\left(S_{j}^{1}(k)\right) \leq v_{I I}\left(S_{j}\right)$. This completes the proof.

With the exception of the case $p=\infty$, we do not know yet whether $C\left(N, v_{I I}\right)$ is nonempty for all $\ell_{p}$ metric spaces over $\mathbb{R}^{\mathrm{d}}$. We assume without loss of generality that $v_{i} \neq v_{0}$ for all $i=1, \ldots, n$.

Theorem 5.4 The core of the game $\left(N, v_{I I}\right)$, defined on the $\ell_{\infty}$ metric space over $\mathbb{R}^{\mathrm{d}}$, is nonempty. Specifically, $C\left(N, v_{I}\right)=C\left(N, v_{I I}\right)$.

Moreover, if $D\left(N_{0}\right)=d\left(v_{0}, v_{j}\right)$, for some $v_{j} \in N$, the dimension of $C\left(N, v_{I I}\right)$ is $n-1$, and there is $x^{*} \in C\left(N, v_{I I}\right)$ such that $x_{t}^{*}>0$, for any $v_{t} \in N$. Also, if $D\left(N_{0}\right)=d\left(v_{i}, v_{j}\right)$, for some $v_{i}, v_{j} \in N$, and $d\left(v_{i}, v_{j}\right)<d\left(v_{i}, v_{0}\right)+d\left(v_{j}, v_{0}\right)$, then the dimension of $C\left(N, v_{I I}\right)$ is $n-1$, and there is $x^{*} \in C\left(N, v_{I I}\right)$ such that $x_{t}^{*}>0$, for any $v_{t} \in N$.

Proof. When $p=\infty$, it is easy to see that for any set $S$ we have $v_{I}(S)=D\left(S \cup\left\{v_{0}\right\}\right)=$ $2 R\left(S \cup\left\{v_{0}\right\}\right)=v_{I I}(S)$. Thus, $C\left(N, v_{I}\right)=C\left(N, v_{I I}\right)$, and the nonemptiness of the core follows from Remark 3.3.

Suppose without loss of generality that $D\left(N_{0}\right)=d\left(v_{0}, v_{1}\right)$. Let $\alpha=\left(\alpha_{1}, \alpha_{2}, \ldots, \alpha_{n}\right)$ be an arbitrary real vector satisfying $0 \leq \alpha_{1} \leq \min _{t=1, \ldots, n} d\left(v_{0}, v_{t}\right), \alpha_{1}=\sum_{j=2}^{n} \alpha_{j}$ and $\alpha_{j} \geq 0$, $j=2, \ldots, n$.

We show that the allocation $x^{\alpha}=\left(d\left(v_{0}, v_{1}\right)-\alpha_{1}, \alpha_{2}, \ldots, \alpha_{n}\right)$ is in $C\left(N, v_{I I}\right)$. First, by definition $x^{\alpha}(N)=d\left(v_{0}, v_{1}\right)=D\left(N_{0}\right)=v_{I I}(N)$. Next consider a coalition $S \subseteq N$. If $v_{1} \in S$, then $x^{\alpha}(S) \leq d\left(v_{0}, v_{1}\right) \leq v_{I I}(S)$. If $v_{1} \neq S$, then $x^{\alpha}(S) \leq \alpha_{1} \leq \min _{t=1, \ldots, n} d\left(v_{0}, v_{t}\right) \leq v_{I I}(S)$.

To see that the dimension of $C\left(N, v_{I I}\right)$ in this case is $n-1$, let $\epsilon$ be a sufficiently small positive real, and consider the $n-1$ independent core allocations $\left\{x^{\alpha(q)}\right\}, q=2, \ldots, n$, where $\alpha(q)$ is the vector defined by $\alpha_{1}(q)=\epsilon, \alpha_{q}(q)=\epsilon$, and $\alpha_{t}(q)=0$, for any $t=2, \ldots, n ; t \neq q$. The allocation $x^{*}=\sum_{q=2}^{n} x^{\alpha(q)} /(n-1)$ is in the core and has strictly positive components.

Next, suppose without loss of generality that $D\left(N_{0}\right)=d\left(v_{1}, v_{2}\right)$ and $d\left(v_{1}, v_{2}\right)<d\left(v_{0}, v_{1}\right)+$ $d\left(v_{0}, v_{2}\right)$. Let $\delta_{1}, \delta_{2}$ be a pair of positive reals satisfying $0<\delta_{1}<d\left(v_{0}, v_{1}\right), 0<\delta_{2}<d\left(v_{0}, v_{2}\right)$, and $\delta_{1}+\delta_{2}=d\left(v_{1}, v_{0}\right)+d\left(v_{2}, v_{0}\right)-d\left(v_{1}, v_{2}\right)$.

Let $\alpha=\left(\alpha_{1}, \alpha_{2}, \ldots, \alpha_{n}\right)$ be an arbitrary real vector satisfying $\alpha_{1} \leq d\left(v_{1}, v_{0}\right)-\delta_{1}, \alpha_{2} \leq$ $d\left(v_{2}, v_{0}\right)-\delta_{2}, 0 \leq \alpha_{1}+\alpha_{2} \leq \min _{t=1, \ldots, n} d\left(v_{0}, v_{t}\right), 0 \leq \alpha_{1}+\alpha_{2} \leq \min \left\{\delta_{1}, \delta_{2}\right\}, \alpha_{1}+\alpha_{2}=\sum_{j=3}^{n} \alpha_{j}$ and $\alpha_{j} \geq 0, j=1, \ldots, n$. 
We show that the allocation

$$
x^{\alpha}=\left(d\left(v_{0}, v_{1}\right)-\delta_{1}-\alpha_{1}, d\left(v_{0}, v_{2}\right)-\delta_{2}-\alpha_{2}, \alpha_{3}, \ldots, \alpha_{n}\right)
$$

is in $C\left(N, v_{I I}\right)$. First, by definition $x^{\alpha}(N)=d\left(v_{1}, v_{2}\right)=D\left(N_{0}\right)=v_{I I}(N)$. Next consider a coalition $S \subseteq N$. If $v_{1}, v_{2} \in S$, then $x^{\alpha}(S) \leq d\left(v_{1}, v_{2}\right)=v_{I I}(S)$. If $v_{1} \in S, v_{2} \neq S$, then $x^{\alpha}(S) \leq d\left(v_{1}, v_{0}\right)-\delta_{1}-\alpha_{1}+\sum_{q=3}^{n} \alpha_{q} \leq d\left(v_{1}, v_{0}\right)-\alpha_{2}-\alpha_{1}+\sum_{q=3}^{n} \alpha_{q} \leq d\left(v_{1}, v_{0}\right) \leq v_{I I}(S)$. Similarly, if $v_{1} \neq S, v_{2} \in S$, we obtain $x^{\alpha}(S) \leq d\left(v_{2}, v_{0}\right) \leq v_{I I}(S)$. Finally, suppose that $v_{1}, v_{2} \neq S$. Then, $x^{\alpha}(S) \leq \alpha_{1}+\alpha_{2} \leq \min _{t=1, \ldots, n} d\left(v_{0}, v_{t}\right) \leq v_{I I}(S)$.

To see that the dimension of $C\left(N, v_{I I}\right)$ in this case is $n-1$, let $\epsilon$ be a sufficiently small positive real, and consider the collection of $n-2$ independent core allocations $\left\{x^{\alpha(q)}\right\}, q=$ $3, \ldots, n$, where $\alpha(q)$ is the vector defined by $\alpha_{1}(q)=\epsilon, \alpha_{q}(q)=\epsilon$, and $\alpha_{t}(q)=0$, for any $t=2, \ldots, n ; t \neq q$. Add to this collection the allocation $x^{\alpha(2)}$, where $\alpha(2)$ is the vector defined by $\alpha_{2}(q)=\epsilon, \alpha_{3}(q)=\epsilon$, and $\alpha_{t}(q)=0$, for any $t=1, \ldots, n, t \neq 2,3$. The allocation $x^{*}=\sum_{q=2}^{n} x^{\alpha(q)} /(n-1)$ is in the core and has strictly positive components. This completes the proof.

Augmenting the result in the last theorem, the next example illustrates that when the conditions in the theorem are not satisfied, the dimension of the core can even be zero. Specifically, for any number of players, even in the $\ell_{\infty}$ planar case, the core can be a singleton where only two players share the total cost, in spite of the fact that the distance from each player to the server $v_{0}$ is positive.

Example 5.5 Consider the set of points $N_{0}=\left\{v_{0}, v_{1}, \ldots, v_{k}\right\}$ where $v_{0}=(0,0), v_{1}=(0,1)$, $v_{2}=(0,-1), v_{3}=(1,0)$ and $v_{i}=\left(a_{i}, 0\right), 0<a_{i}<1$, for $i=4,5, \ldots, k$. Since $v_{I}(S)=2$, if $\left\{v_{1}, v_{2}\right\} \subseteq N$, and $v_{I}(S) \leq 1$, otherwise, it is easy to see that $C\left(N, v_{I}\right)=C\left(N, v_{I I}\right)=$ $\{(1,1,0, \ldots, 0)\}$.

Corollary 5.1 The core of the game $\left(N, v_{I I}\right)$, defined on the $\ell_{1}$ metric plane is nonempty. Specifically, $C\left(N, v_{I}\right)=C\left(N, v_{I I}\right)$.

Proof. Since the rectilinear norm, $\ell_{1}$, is equivalent to the $\ell_{\infty}$ norm on the plane, for any subset $S, D\left(S \cup\left\{v_{0}\right\}\right)=2 R\left(S \cup\left\{v_{0}\right\}\right)$ for the rectilinear planar case. Therefore, the core of the respective minimum radius game in the plane is nonempty.

\section{$5.1 \quad$ Euclidean spaces}

Turning to the Euclidean case, in general, the equality $v_{I}(N)=v_{I I}(N)$ may not hold even in the planar case. From Proposition 2.1 it follows that the characteristic function $v_{I I}(S)$ is subadditive also for the Euclidean model. However, it does not follow from the general analysis in previous sections that the core of the Euclidean planar game is nonempty. 
In spite of that, we will prove that $C\left(N, v_{I I}\right)$ is nonempty for the Euclidean planar case. More specifically, there is a core allocation where at most 3 players (points) pay positive amounts. These are points defining $C(V)$, the minimal circle in the plane enclosing the set $V$.

Theorem 5.5 The core $C\left(N, v_{I I}\right)$ of the minimal radius location game $\left(N, v_{I I}\right)$ in the Euclidean planar case is non-empty.

Proof. See the Appendix.

Remark 5.1 In the minimum radius location game $\left(N, v_{I I}\right)$, for each coalition $S, v_{I I}$ is defined as twice the solution value to the 1-center problem for the set of nodes $S \cup\left\{v_{0}\right\}$. Similarly we can consider location games defined by other common optimization criteria often used in facility location models. For example, consider the minimum median location game, $\left(N, v_{I I I}\right)$, where for each coalition $S, v_{I I I}$ is defined as the solution value to the 1-median problem for the set of nodes $S \cup\left\{v_{0}\right\}$.

We note that from the cooperative point of view the above definition does not even induce the desirable property of subadditivity. Thus, players may not even have the incentive to cooperate, as shown in the following example.

Example 5.6 Consider a 4-node path with the edge set $E=\left\{\left(v_{1}, v_{0}\right),\left(v_{0}, v_{2}\right),\left(v_{2}, v_{3}\right)\right\}$. Edges are of unit length. It is easy to see that $v_{I I I}(N)=4, v_{I I I}\left(\left\{v_{1}\right\}\right)=1$ and $v_{I I I}\left(\left\{v_{2}, v_{3}\right\}\right)=$ 2. Hence, $v_{I I I}\left(\left\{v_{1}\right\}\right)+v_{I I I}\left(\left\{v_{2}, v_{3}\right\}\right)=3<4=v_{I I I}(N)$. The core is empty in this example since the set of constraints, $x_{1} \leq 1, x_{2}+x_{3} \leq 2$ and $x_{1}+x_{2}+x_{3}=4$ is inconsistent.

Two different median related cooperative games where subadditivity is ensured by introducing set up costs can be found in Puerto et al. (2001) and Mallozi (2011).

\section{Conclusions and open problems}

In this paper we have introduced a new class of cooperative location games, the Minimum Radius Location Game (MRLG). In such a game the characteristic function is defined as the radius of each coalition, including a distinguished point that can be viewed as a server. Motivated by potential applications, we have focused mainly on the important cases of network metric spaces and the $\ell_{p}$-normed spaces over $\mathbb{R}^{\mathrm{d}}$. For these spaces we give complete polyhedral characterizations of the core of the MRLG by using only a polynomial number of linear inequalities. Using these characterizations, emptiness of the core can be tested efficiently by linear programming algorithms.

We have shown that, in general, the core of the MRLG might be empty even for geometric planar road networks with Euclidean distances. In contrast, we have proved that for the 
Euclidean normed plane the core is always nonempty. Moreover, we have constructed a core allocation in which at most three players pay positive costs. These players correspond to the demand points defining the minimal circle enclosing all the demand points and the server.

With the exception of the planar Euclidean case and the $\ell_{\infty}$ space, for any $d \geq 1$, it is still unknown whether the MRLG has a nonempty core for any $\ell_{p}$ space.

We have also given some sufficient conditions for the core to be nonempty. These conditions are based on the relationship between the MRLG and the MDLG, which always has some core allocation. Similar to the Euclidean planar case, in the core allocations that we construct only a pair of players share the total cost. Although practically allocations where only a very small number of players pay some positive cost, may not be easy to implement, their main role is just to establish the nonemptiness of the core. As illustrated in Section 5, in some cases the core itself is a singleton, where only two players share the total cost. If the core is not a singleton of the above type, then to get an allocation in the core which is more "acceptable", one can use linear programming methods on the aforementioned efficient characterizations of the core. For example, to find a core allocation where each player $v_{i}$, $v_{i} \neq v_{0}$, shares some positive part of $v_{I I}(N)$, we can consider the problem of finding a core allocation which will maximize the minimum pay over all these players. The latter can be formulated as a linear program over the core.

We conclude with a few more open questions. First, it is still unclear what general properties of the radius game will at least unify the nonemptiness results presented here, e.g., for median graphs and the Euclidean plane. Second, we have demonstrated that the core can be empty for simple geometric network spaces, where several nodes, different from the server, are not players. Is the core always nonempty when there are no such nodes, i.e., when the game is complete? Finally, in spite of our efforts we have not been able to find a shorter and more elegant proof of the nonemptiness of the core of the MRLG in the planar Euclidean case, using general tools from cooperative game theory, e.g., Bondareva-Shapley conditions, or equivalently linear programming duality. Is there such a proof?

\section{References}

Bird C.G., 1976. On cost allocation for a spanning Tree: A game theoretic approach. Networks 6, 335-350.

Caprara, A., Letchford, A. N., 2010. New techniques for cost sharing in combinatorial optimization games. Mathematical Programming 124, 93-118.

Faigle, U., Kern, W., Fekete, S.P., Hochstattler, W., 1997. On the complexity of testing membership in the core of min-cost spanning tree games. International Journal of Game Theory 26, 361-366. 
Granot, D., Huberman, G., 1981. Minimum cost spanning tree games. Mathematical Programming 21, 1-18.

Granot, D., Huberman, G., 1984. On the core and nucleolus of minimum cost spanning tree games. Mathematical Programming 29, 323-347.

Handler, G., 1973. Minimax location of a facility in an undirected tree graph. Transportation Science 7, 287-293.

Hassin, R., Tamir, A., 1995. On the minimum diameter spanning tree problem. Information Processing Letters 53, 109-111.

Liu, Y., Huang, J., 2009. Diameter-preserving spanning trees in sparse weighted graphs. Graphs and Combinatorics 25, 753-758.

Mallozi, L., 2011. Cooperative games in facility location situations with regional fixed costs. Optimization Letters 5, 171-183.

Megiddo, N., 1978. Computational complexity of the game theory approach to cost allocation for a tree. Mathematics of Operations Research 3, 189-196.

Mulder, H., 1978. The structure of median graphs. Discrete Mathematics 25, 197-204.

Mulder, H., 1980. The interval function of a graph. Vol. 132. Math. Center Tracts.

Papadopoulos, A., 2005. Metric Spaces, Convexity and Nonpositive Curvature. European Mathematical Society.

Puerto, J., García-Jurado, I., Fernández, F., 2001. On the core of a class of location games. Mathematical Methods of Operations Research 54 (3), 373-385.

Puerto, J., Tamir, A., Perea, F., 2010. Cooperative location games based on the minimmum diameter spanning steiner subgraph problem. Prepublicaciones Facultad Matemáticas, Universidad de Sevilla.

Tamir, A., 1991. On the core of network synthesis games. Mathematical Programming 50, 123-135.

Tardos, E., 1986. A strongly polynomial algorithm to solve combinatorial linear programs. Operations Research 34, 250-256.

Zurcher, S., 2007. Smallest enclosing ball for a point set with strictly convex level sets. Masters Thesis, Institute of Theoretical Computer Science, ETH Zurich. 


\section{Appendix: Proof of Theorem 5.5}

First of all, note that we have not been able to produce a short existence proof of core allocations, based on known general theorems in cooperative game theory. Instead, our proof is based on a long case analysis.

We divide the proof analyzing three exhaustive cases depending on the relative position of $C(V)$ (the minimum circle in the plane enclosing $V$ ):

i) $C(V)$ is determined by two points of $V$ (Proposition 7.1).

ii) $C(V)$ is determined by three points of $V$ and $v_{0}$ is one of them (Proposition 7.2).

iii) $C(V)$ is determined by three points and $v_{0}$ is not among them (Proposition 7.3).

Remark 7.1 Our proof is based on showing that the core of the subgame defined by at most three players, say $\left\{v_{1}, v_{2}, v_{3}\right\}$, corresponding to the points defining $C(V)$ is nonempty. Of course, the latter subgame can be viewed as a 3-player game on a complete graph $G$ with at most eight nodes. The four extra nodes, augmenting $\left\{v_{0}, v_{1}, v_{2}, v_{3}\right\}$, are those representing the centers of the minimal circles enclosing the four triplets $\left\{\left(v_{0}, v_{1}, v_{2}\right),\left(v_{0}, v_{1}, v_{3}\right)\right.$, $\left.\left(v_{0}, v_{2}, v_{3}\right),\left(v_{1}, v_{2}, v_{3}\right)\right\}$, respectively. The edge lengths of $G$, inducing the respective space $A(G)$, are the Euclidean distances between the respective pairs of points representing the edges.

Proposition 7.1 If the minimal circle enclosing $V$ is determined by two points in $V$, then $C\left(N, v_{I I}\right) \neq \emptyset$.

Proof. We observe that in this case, $D(V)=2 R(V)$ since the two points are diametrical. Hence, $v_{I}(N)=v_{I I}(N)$, and the result follows from Remark 3.3.

Next, suppose that $C(V)$ is determined by the points in $V^{\prime}=\left\{v_{i_{1}}, v_{i_{2}}, v_{i_{3}}\right\}$. Specifically, if $r^{*}=R(V)$ is the radius of this circle, then the radius of $C\left(V^{\prime}\right)$, the minimal circle enclosing $V^{\prime}$ is also $r^{*}$. Without loss of generality assume that $V^{\prime}=\left\{v_{1}, v_{2}, v_{i_{3}}\right\}$, where $v_{i_{3}}=v_{0}$ or $v_{i_{3}}=v_{3}$, depending on cases (ii) and (iii) above.

Proposition 7.2 Suppose that $C(V)$ is determined by the points $V^{\prime}=\left\{v_{1}, v_{2}, v_{i_{3}}\right\}, v_{i_{3}}=v_{0}$, and $d\left(v_{1}, v_{0}\right) \geq d\left(v_{2}, v_{0}\right)$. Then, the allocation defined by $x_{1}=d\left(v_{1}, v_{0}\right), x_{2}=2 r^{*}-d\left(v_{1}, v_{0}\right)$, and $x_{i}=0$, for $i \neq 1,2$, is in $C\left(N, v_{I I}\right)$, where $r^{*}=R(V)$.

Proof. Let $v_{I I}(N)=2 R(V)=2 r^{*}$ and

$$
r^{\prime}=\left(d\left(v_{1}, v_{0}\right)+d\left(v_{2}, v_{0}\right)\right) / 2 .
$$

We claim that $r^{*} \leq r^{\prime}$. Indeed, consider a point $x^{\prime}$ on the segment $\left[v_{1}, v_{0}\right]$ satisfying $d\left(x^{\prime}, v_{1}\right)=r^{\prime}$. Then, from the triangle inequality, $d\left(x^{\prime}, v_{2}\right) \leq d\left(x^{\prime}, v_{0}\right)+d\left(v_{0}, v_{2}\right)=d\left(v_{1}, v_{0}\right)-$ 
$\left(d\left(v_{1}, v_{0}\right)+d\left(v_{2}, v_{0}\right)\right) / 2+d\left(v_{2}, v_{0}\right)=r^{\prime}$. Hence, a circle of radius $r^{\prime}$, centered at the point $x^{\prime}$ on the segment connecting $v_{1}$ and $v_{0}$ and satisfying $d\left(x^{\prime}, v_{1}\right)=r^{\prime}$, encloses the 3 points $\left\{v_{0}, v_{1}, v_{2}\right\}$. Therefore, $r^{*}$, the radius of the minimal circle is at most $r^{\prime}$, and we have $x_{1}=$ $d\left(v_{1}, v_{0}\right)=v_{I I}\left(\left\{v_{1}\right\}\right)$, and $x_{2}=2 r^{*}-d\left(v_{1}, v_{0}\right) \leq 2 r^{\prime}-d\left(v_{1}, v_{0}\right)=d\left(v_{2}, v_{0}\right)=v_{I I}\left(\left\{v_{2}\right\}\right)$. Thus, setting $x_{i}=0$, for $i \neq 1,2$, we obtain an allocation in the core $C\left(N, v_{I I}\right)$.

Next we turn to the allocation of $v_{I I}(N)$ when the three points spanning the minimal circle $C(V)$ are $\left\{v_{1}, v_{2}, v_{3}\right\}$, and $v_{0}$ is inside the circle.

We will need to use some properties of the optimal circle, and the fact that $v_{0}$ is inside. Assume without loss of generality that

$$
d\left(v_{1}, v_{0}\right) \geq d\left(v_{2}, v_{0}\right) \geq d\left(v_{3}, v_{0}\right) .
$$

Remark 7.2 We note that when $v_{0}$ is inside the circle, and no pair of the triplet is diametrical, then in every core allocation for the 3 player game, each player will have to pay some positive amount. (For each pair, $S=\left\{v_{i}, v_{j}\right\} \subseteq\left\{v_{1}, v_{2}, v_{3}\right\}$, we have $x_{i}+x_{j} \leq v_{I I}(S)<2 r^{*}$, implying $x_{k}=2 r^{*}-\left(x_{i}+x_{j}\right)>0$, for $k \neq i, j$.

Next, since the radius of the circle centered at $v_{0}$, and covering the set $\left\{v_{1}, v_{2}, v_{3}\right\}$ is at least $r^{*}$, we must have $d\left(v_{1}, v_{0}\right)=\max \left(d\left(v_{1}, v_{0}\right), d\left(v_{2}, v_{0}\right), d\left(v_{3}, v_{0}\right)\right) \geq r^{*}$. In fact, a stronger inequality holds.

Remark 7.3 Under the assumption on the relative position of the points given in (4), the following inequalities hold:

$$
4 r^{*} \geq d\left(v_{1}, v_{0}\right)+d\left(v_{2}, v_{0}\right) \geq 2 r^{*},
$$

for each point $v_{0}$ in the circle. The left inequality is obvious. To prove the right inequality suppose by contradiction that $2 r^{\prime}=d\left(v_{1}, v_{0}\right)+d\left(v_{2}, v_{0}\right)<2 r^{*}$ for some point $v_{0}$ in the circle. (Recall that $r^{\prime}$ was defined in (3).) Then, a circle of radius $r^{\prime}$, centered at the point $x^{\prime}$ on the segment connecting $v_{1}$ and $v_{0}$ and satisfying $d\left(x^{\prime}, v_{1}\right)=r^{\prime}$, encloses the 4 points $\left\{v_{0}, v_{1}, v_{2}, v_{3}\right\}$ $\left(d\left(v_{1}, x^{\prime}\right)=r^{\prime}\right.$ and for $\left.i=2,3, d\left(v_{i}, x^{\prime}\right) \leq d\left(x^{\prime}, v_{0}\right)+d\left(v_{0}, v_{i}\right) \leq d\left(x^{\prime}, v_{0}\right)+d\left(v_{0}, v_{2}\right)=r^{\prime}.\right)$ Hence, we have contradicted the minimality of $r^{*}$.

Remark 7.4 By maximizing the convex function $d\left(y, v_{1}\right)+d\left(y, v_{2}\right)+d\left(y, v_{3}\right)$, it can be shown that for any point $v_{0}$ inside the enclosing circle we have

$$
2 r^{*} \leq d\left(v_{1}, v_{0}\right)+d\left(v_{2}, v_{0}\right) \leq d\left(v_{1}, v_{0}\right)+d\left(v_{2}, v_{0}\right)+d\left(v_{3}, v_{0}\right) \leq(2+2 \sqrt{2}) r^{*} .
$$

Both, the $2 r^{*}$ uniform lower bound and the $(2+2 \sqrt{2}) r^{*}$ uniform upper bound are asymptotically tight. (For the lower bound consider the case where $d\left(v_{1}, v_{0}\right)=2 r^{*}$, and $v_{2}$ and 
$v_{3}$ are arbitrarily close to $v_{0}$. For the upper bound consider the case where $v_{1}$ and $v_{0}$ are the end points of some diameter, say $d_{1}$, and $v_{2}$ and $v_{3}$ are the end points of the diameter perpendicular to $d_{1}$.)

Another useful observation is that for any triplet $\left\{v_{0}, v_{i}, v_{j}\right\}, v_{I I}\left(\left\{v_{i}, v_{j}\right\}\right)$ is bounded below by the longest edge of the triangle formed by the triplet,

$$
v_{I I}\left(\left\{v_{i}, v_{j}\right\}\right) \geq \max \left(d\left(v_{i}, v_{j}\right), d\left(v_{i}, v_{0}\right), d\left(v_{j}, v_{0}\right)\right) .
$$

Moreover, if the longest edge is not the diameter, then $v_{I I}\left(\left\{v_{i}, v_{j}\right\}\right)=2 r$, where

$$
\begin{aligned}
& r=a b c / 4 \sqrt{k(k-a)(k-b)(k-c)}, \\
& k=(a+b+c) / 2, a=d\left(v_{i}, v_{0}\right), b=d\left(v_{j}, v_{0}\right), c=d\left(v_{i}, v_{j}\right) .
\end{aligned}
$$

We will need the following lemma.

Lemma 7.1 Given a triplet of points $v_{1}^{\prime}, v_{2}^{\prime}, v_{3}^{\prime}$, let $r^{*}$ be the radius of the minimal disk enclosing the triplet, and suppose that $2 r^{*}>d\left(v_{i}^{\prime}, v_{j}^{\prime}\right)$, for all $i, j=1,2,3$. Given a positive real number $r^{\prime}$, such that $d\left(v_{3}^{\prime},\left(v_{1}^{\prime}+v_{2}^{\prime}\right) / 2\right) \geq r^{\prime}$, let $u^{\prime}$ be a point in the above disk satisfying $d\left(v_{3}^{\prime}, u^{\prime}\right)=r^{\prime}$. Then, $v^{1,2}\left(u^{\prime}, r^{\prime}\right)$, the diameter of the smallest circle enclosing $v_{1}^{\prime}, v_{2}^{\prime}$ and $u^{\prime}$, satisfies $v^{1,2}\left(u^{\prime}, r^{\prime}\right) \geq 2 r^{*}-r^{\prime}$.

Proof. We assume without loss of generality that $r^{*}=1$ and $d\left(v_{1}^{\prime}, v_{3}^{\prime}\right) \geq d\left(v_{2}^{\prime}, v_{3}^{\prime}\right)$. Therefore, the three points admit a representation as $v_{1}^{\prime}=(-\cos \alpha,-\sin \alpha), v_{2}^{\prime}=(\cos \alpha,-\sin \alpha)$ and $v_{3}^{\prime}=(\cos \beta, \sin \beta)$ with $0 \leq \alpha \leq \beta \leq \pi / 2$.

The supposition $2 r^{*}=2>d\left(v_{i}^{\prime}, v_{j}^{\prime}\right)$, for all $i, j=1,2,3$, also implies that $\alpha<\beta$.

With this configuration, $(0,0)$ is the center of the ball that spans $v_{1}^{\prime}, v_{2}^{\prime}, v_{3}^{\prime}$. (Note that $r^{*}=1=\left\|v_{i}^{\prime}\right\|, i=1,2,3$.)

Define $B\left(v_{3}^{\prime}, r^{\prime}\right)$ to be the ball centered at $v_{3}^{\prime}$ with radius $r^{\prime}$. Let $B^{*}=B^{*}\left(v_{1}^{\prime}, v_{2}^{\prime}, r^{\prime}\right)$ be the ball of smallest radius which contains $v_{1}^{\prime}, v_{2}^{\prime}$ and intersects the boundary of $B\left(v_{3}^{\prime}, r^{\prime}\right) . r\left(B^{*}\right)$ denotes the radius of $B^{*}$. See Figure 5 for a graphical instance of this situation. It is clearly sufficient to show that $2 r\left(B^{*}\right) \geq 2-r^{\prime}$.

We first prove that $c^{\prime}$, the center of $B^{*}$, satisfies $c^{\prime}=(0, c)$, (i.e., $c^{\prime}$ is on the bisector of $v_{1}^{\prime}$ and $v_{2}^{\prime}$ ) with $c<0$. Since $r^{\prime}>0$ it follows that $r\left(B^{*}\right)<r^{*}=1$. Thus, if $c^{\prime}=(0, c)$, then clearly $c<0$.

If the edge $\left[v_{1}^{\prime}, v_{2}^{\prime}\right]$ intersects $B\left(v_{3}^{\prime}, r^{\prime}\right)$, then $c^{\prime}$ is the midpoint of $\left[v_{1}^{\prime}, v_{2}^{\prime}\right]$ and $c^{\prime}=(0,-\sin \alpha)$. Hence, suppose that $B\left(v_{3}^{\prime}, r^{\prime}\right)$ does not intersect $\left[v_{1}^{\prime}, v_{2}^{\prime}\right]$. For $i=1,2$, let $u_{i}$ be the point on the edge $\left[v_{3}^{\prime}, v_{i}^{\prime}\right]$ such that $d\left(u_{i}, v_{3}^{\prime}\right)=r^{\prime}$. The ball $B^{*}$ is determined by the triplet $\left\{v_{1}^{\prime}, v_{2}^{\prime}, u\right\}$, where $u$ is some point on the arc of $B\left(v_{3}^{\prime}, r^{\prime}\right)$, connecting $u_{1}$ with $u_{2}$. 


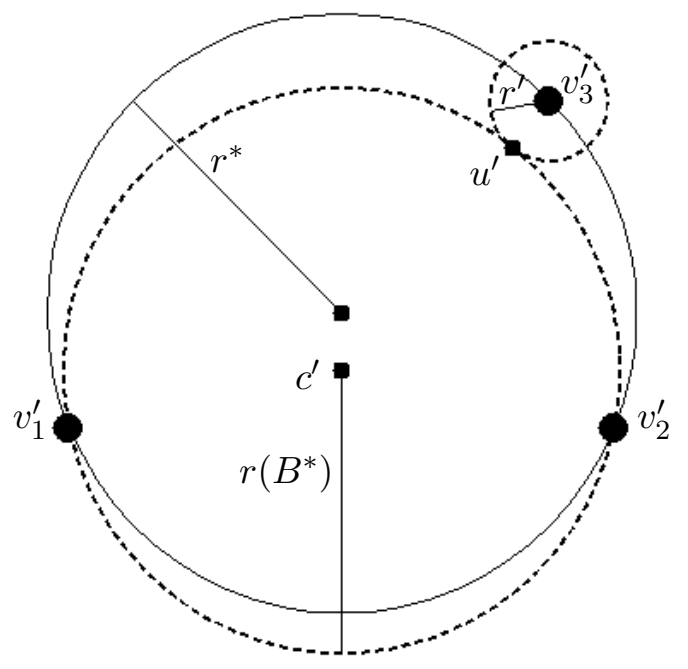

Figure 5: An instance of the situation expressed in Lemma 7.1.

From the minimality of $B^{*}$, if all the three points are on the boundary of $B^{*}$, then clearly $c^{\prime}$ is on the bisector of $v_{1}^{\prime}$ and $v_{2}^{\prime}$ with $c<0$. The same result holds when $\left[v_{1}^{\prime}, v_{2}^{\prime}\right]$ is a diameter of $B^{*}$.

Hence, suppose that either $\left[u, v_{2}^{\prime}\right]$ is a diameter of $B^{*}$, and $v_{1}^{\prime}$ is strictly inside $B^{*}$, or $\left[u, v_{1}^{\prime}\right]$ is a diameter of $B^{*}$, and $v_{2}^{\prime}$ is strictly inside $B^{*}$.

In the former case we must have $u=u_{2}$, otherwise, by replacing $u$ by $u$ ", sufficiently close to $u$, on the arc of $B\left(v_{3}^{\prime}, r^{\prime}\right)$, connecting $u$ and $u_{2}$, we would obtain a ball containing $v_{1}^{\prime}, v_{2}^{\prime}$ and $u$ ", whose radius is smaller than $r\left(B^{*}\right)$. But, $\left[v_{2}^{\prime}, u_{2}\right]$ cannot be a diameter of $B^{*}$, since $d\left(v_{1}^{\prime}, u_{2}\right)>d\left(v_{2}^{\prime}, u_{2}\right)$.

Finally, suppose that $\left[u, v_{1}^{\prime}\right]$ is a diameter of $B^{*}$, and $v_{2}^{\prime}$ is strictly inside $B^{*}$. Again, by the above argument we must have $u=u_{1}$. We will show that the midpoint of $\left[v_{1}^{\prime}, u_{1}\right]$ cannot be the center of $B^{*}$.

Let $c^{u}=\left(c^{1}, c^{2}\right)$ be the midpoint of $\left[v_{1}^{\prime}, u_{1}\right]$. Define $\bar{c}=\left(0, c^{2}\right)$. Note that $c^{1} \leq$ $\frac{-\cos \alpha+\cos \beta}{2}<0$, since $\alpha<\beta$. Then, it is easy to see that

$$
r\left(B^{*}\right)=d\left(v_{1}^{\prime}, c^{u}\right)<d\left(v_{1}^{\prime}, \bar{c}\right)=d\left(v_{2}^{\prime}, \bar{c}\right)<d\left(v_{2}^{\prime}, c^{u}\right) .
$$

In particular, we obtain the contradiction that $v_{2}^{\prime}$ is outside $B^{*}$.

This concludes the proof that $c^{\prime}=(0, c)$ for some $c<0$.

\section{$\underline{\text { Claim. }}$}

$$
d\left(v_{3}^{\prime}, c^{\prime}\right)+d\left(c^{\prime}, v_{2}^{\prime}\right)>d\left(v_{3}^{\prime}, 0\right)+d\left(0, v_{2}^{\prime}\right)=2 .
$$


Proving the claim will conclude the proof of the lemma since

$$
2 r^{*}-r^{\prime}=2-r^{\prime}=d\left(v_{3}^{\prime}, 0\right)+d\left(0, v_{2}^{\prime}\right)-r^{\prime}<d\left(v_{3}^{\prime}, c^{\prime}\right)+d\left(c^{\prime}, v_{2}^{\prime}\right)-r^{\prime} \leq v^{1,2}\left(u, r^{\prime}\right) .
$$

(The last inequality follows from the triangle inequality $d\left(v_{3}^{\prime}, c^{\prime}\right) \leq d\left(v_{3}^{\prime}, u\right)+d\left(u, c^{\prime}\right)=$ $r^{\prime}+d\left(u, c^{\prime}\right)$, and the fact that $\left.d\left(c^{\prime}, u\right)=d\left(c^{\prime}, v_{2}^{\prime}\right)=v^{1,2}\left(u, r^{\prime}\right) / 2.\right)$

Proof of claim. Consider the function $f(z)=\left\|z-v_{2}^{\prime}\right\|+\left\|z-v_{3}^{\prime}\right\|$ when $z$ moves on the line $\left\{z \in \mathbb{R}^{2}: z_{1}=0\right\}$. Parametrizing the function on its unique variable, say $\lambda$, results in:

$$
\begin{aligned}
f(\lambda) & =\sqrt{\cos ^{2} \alpha+(\lambda+\sin \alpha)^{2}}+\sqrt{\cos ^{2} \beta+(\lambda-\sin \beta)^{2}} \\
& =\sqrt{1+\lambda^{2}+2 \lambda \sin \alpha}+\sqrt{1+\lambda^{2}-2 \lambda \sin \beta}, \quad \lambda \leq 0 .
\end{aligned}
$$

Proving (5) is equivalent to showing that $f(c)>f(0)$. Then, observe that $f$ is a real value convex function and thus if the function is decreasing at 0 the claim is true.

To this end, we compute the derivative of $f$ at 0 .

$$
f^{\prime}(\lambda)=\frac{\lambda+\sin \alpha}{\sqrt{1+\lambda^{2}+2 \lambda \sin \alpha}}+\frac{\lambda-\sin \beta}{\sqrt{1+\lambda^{2}-2 \lambda \sin \beta}}
$$

and substituting at 0 , we get

$$
f^{\prime}(0)=\sin \alpha-\sin \beta<0,
$$

since $\alpha<\beta$. This concludes the proof of the claim.

One can give necessary conditions for the existence of a core allocation $x=\left(x_{1}, x_{2}, x_{3}\right)$. For example, since $x_{1}+x_{2}+x_{3}=2 r^{*}$, and $x_{3} \leq d\left(v_{3}, v_{0}\right)=v_{I I}\left(\left\{v_{3}\right\}\right)$, we must have $v_{I I}\left(\left\{v_{1}, v_{2}\right\}\right) \geq x_{1}+x_{2}=2 r^{*}-x_{3} \geq 2 r^{*}-d\left(v_{3}, v_{0}\right)$. The next lemma gives a sharper condition.

Lemma 7.2 Given a triplet of points $v_{1}^{\prime}, v_{2}^{\prime}, v_{3}^{\prime}$, let $r^{*}$ be the radius of the minimal circle enclosing the triplet. Let $v_{0}$ be a point inside this minimal circle. Suppose that $2 r^{*}>d\left(v_{i}^{\prime}, v_{j}^{\prime}\right)$, for all $i, j=1,2,3$.

Then, $d\left(v_{3}^{\prime},\left(v_{1}^{\prime}+v_{2}^{\prime}\right) / 2\right)>d\left(v_{1}^{\prime}, v_{2}^{\prime}\right) / 2$, and $v_{I I}\left(\left\{v_{1}^{\prime}, v_{2}^{\prime}\right)\right\}$, defined as the diameter of the minimal circle containing $\left\{v_{0}, v_{1}^{\prime}, v_{2}^{\prime}\right\}$, satisfies $2 r^{*}-d\left(v_{0}, v_{3}^{\prime}\right) \leq v_{I I}\left(\left\{v_{1}^{\prime}, v_{2}^{\prime}\right)\right\}$.

\section{Proof.}

Suppose without loss of generality that $r^{*}=1$ and $d\left(v_{1}^{\prime}, v_{3}^{\prime}\right) \geq d\left(v_{2}^{\prime}, v_{3}^{\prime}\right)$. We introduce a reference system of coordinates with the origin $(0,0)$ at $C$, the center of the circle spanned by $v_{1}^{\prime}, v_{2}^{\prime}, v_{3}^{\prime}$. Then, $v_{1}^{\prime}=(-\cos \alpha,-\sin \alpha), v_{2}^{\prime}=(\cos \alpha,-\sin \alpha)$ and $v_{3}^{\prime}=(\cos \beta, \sin \beta)$ with $0<\alpha<\beta \leq \pi / 2$. 
For any feasible $v_{3}^{\prime}(\beta)=(\cos \beta, \sin \beta), \pi / 2 \geq \beta>\alpha>0$, we have

$$
d\left(\left(v_{1}^{\prime}+v_{2}^{\prime}\right) / 2, v_{3}^{\prime}(\beta)\right)=\sqrt{1+\sin ^{2} \alpha+2 \sin \beta \sin \alpha} \geq \sqrt{1+3 \sin ^{2} \alpha}>1>d\left(v_{1}^{\prime}, v_{2}^{\prime}\right) / 2 .
$$

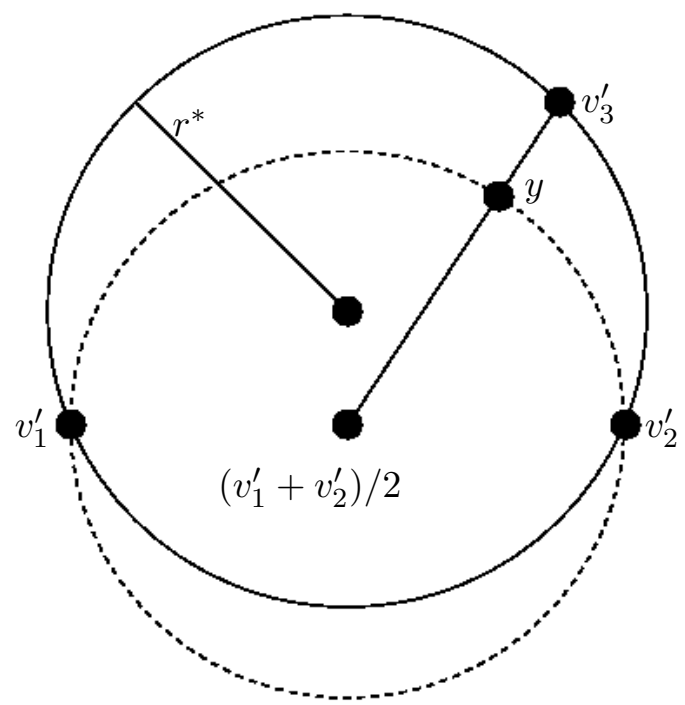

Figure 6: An instance of the situation expressed in Lemma 7.2.

Let $\left.r^{\prime \prime}=d\left(v_{3}^{\prime},\left(v_{1}^{\prime}+v_{2}^{\prime}\right) / 2\right)\right)-d\left(v_{1}^{\prime}, v_{2}^{\prime}\right) / 2$. $\left(r^{\prime \prime}\right.$ is well defined since $d\left(v_{1}^{\prime}, v_{2}^{\prime}\right) / 2 \leq d\left(v_{3}^{\prime},\left(v_{1}^{\prime}+\right.\right.$ $\left.\left.\left.v_{2}^{\prime}\right) / 2\right)\right)$.) Let $y$ be the point on the line segment $\left.\left[v_{3}^{\prime},\left(v_{1}^{\prime}+v_{2}^{\prime}\right) / 2\right)\right]$, satisfying $d\left(y, v_{3}^{\prime}\right)=r^{\prime \prime}$.

Note that the minimal circle enclosing $\left\{y, v_{1}^{\prime}, v_{2}^{\prime}\right\}$ is centered at the point $\left(v_{1}^{\prime}+v_{2}^{\prime}\right) / 2$, and its diameter is $d\left(v_{1}^{\prime}, v_{2}^{\prime}\right)$.

Applying Lemma 7.1 with $r^{\prime}=r^{\prime \prime}$ and $u=y$, we obtain

$$
2-d\left(v_{3}^{\prime}, y\right)=2-r^{\prime \prime} \leq v^{12}\left(y, r^{\prime \prime}\right)=d\left(v_{1}^{\prime}, v_{2}^{\prime}\right)
$$

We consider two cases:

i) $d\left(v_{3}^{\prime}, v_{0}\right) \leq d\left(y, v_{3}^{\prime}\right)$.

In this case we apply Lemma 7.1 with $r^{\prime}=d\left(v_{3}^{\prime}, v_{0}\right)$ and $u=v_{0}$. (Note that in this case $\left.r^{\prime} \leq d\left(v_{3}^{\prime}, y\right) \leq d\left(v_{3}^{\prime},\left(v_{1}^{\prime}+v_{2}^{\prime}\right) / 2\right).\right)$ Thus,

$$
2-r^{\prime}=2-d\left(v_{3}^{\prime}, v_{0}\right) \leq v^{12}\left(v_{0}, r^{\prime}\right)=v_{I I}\left(\left\{v_{1}^{\prime}, v_{2}^{\prime}\right\}\right)
$$

ii) $d\left(v_{3}^{\prime}, v_{0}\right)>d\left(y, v_{3}^{\prime}\right)$.

In this case we have $2-d\left(v_{3}^{\prime}, v_{0}\right)<2-d\left(y, v_{3}^{\prime}\right)$. Applying (6) we obtain

$$
2-d\left(v_{3}^{\prime}, v_{0}\right)<2-d\left(y, v_{3}^{\prime}\right) \leq d\left(v_{1}^{\prime}, v_{2}^{\prime}\right) \leq v_{I I}\left(\left\{v_{1}^{\prime}, v_{2}^{\prime}\right\}\right)
$$


We now continue the proof of the nonemptiness of the core when the minimal circle is defined by the points $\left\{v_{1}, v_{2}, v_{3}\right\}$ and $v_{i} \neq v_{0}$ for all $i=1,2,3$.

\begin{tabular}{|c|c|c|}
\hline & Condition & Allocation \\
\hline Case I & \multicolumn{2}{|c|}{$d\left(v_{i}, v_{j}\right) \geq \sqrt{2}, \forall i, j, i \neq j,\left(d\left(v_{3}, v_{0}\right) \leq d\left(v_{2}, v_{0}\right) \leq d\left(v_{1}, v_{0}\right)\right)$} \\
\hline (a) & $d\left(v_{3}, v_{0}\right) \geq 2-\sqrt{2}$ & $(2 \sqrt{2}-2,2-\sqrt{2}, 2-\sqrt{2})$ \\
\hline (b) & $d\left(v_{3}, v_{0}\right) \leq 2-\sqrt{2}$ & $\left(1-d\left(v_{3}, v_{0}\right) / 2,1-d\left(v_{3}, v_{0}\right) / 2, d\left(v_{3}, v_{0}\right)\right)$ \\
\hline Case II & \multicolumn{2}{|c|}{$\overline{d\left(v_{1}, v_{2}\right)<\sqrt{2} \leq d\left(v_{2}, v_{3}\right) \leq d\left(v_{1}, v_{3}\right)}$} \\
\hline$(\mathrm{c})$ & $d\left(v_{3}, v_{0}\right) \leq \sqrt{2}+\sqrt{2+\sqrt{2}}-2$ & $\left(1-\gamma d\left(v_{3}, v_{0}\right), 1-(1-\gamma) d\left(v_{3}, v_{0}\right), d\left(v_{3}, v_{0}\right)\right)$ \\
\hline (d) & \multicolumn{2}{|c|}{$d\left(v_{3}, v_{0}\right) \geq \sqrt{2}+\sqrt{2+\sqrt{2}}-2$} \\
\hline$(\mathrm{d} .1)$ & \multicolumn{2}{|c|}{$d\left(v_{3}, v_{0}\right) \geq d\left(v_{3},\left(v_{1}+v_{2}\right) / 2\right)-d\left(v_{1}, v_{2}\right) / 2$} \\
\hline $\begin{array}{l}(\mathrm{d} 1 . \mathrm{i}) \\
(\mathrm{d} 1 . \mathrm{ii})\end{array}$ & $\begin{array}{l}d\left(v_{1}, v_{0}\right) \leq d\left(v_{2}, v_{0}\right) \\
d\left(v_{1}, v_{0}\right) \geq d\left(v_{2}, v_{0}\right)\end{array}$ & $\begin{array}{c}\left(m, d\left(v_{1}, v_{2}\right)-m, 2-d\left(v_{1}, v_{2}\right)\right) \\
\left(d\left(v_{1}, v_{2}\right)-m^{\prime}, m^{\prime}, 2-d\left(v_{1}, v_{2}\right)\right)\end{array}$ \\
\hline$(\mathrm{d} .2)$ & \multicolumn{2}{|c|}{$\sqrt{2}+\sqrt{2+\sqrt{2}}-2 \leq d\left(v_{3}, v_{0}\right) \leq d\left(v_{3},\left(v_{1}+v_{2}\right) / 2\right)-d\left(v_{1}, v_{2}\right) / 2$} \\
\hline$(\mathrm{d} 2.1)$ & \multicolumn{2}{|c|}{$d\left(v_{1}, v_{2}\right) \geq 2-d\left(v_{0}, v_{3}\right)$} \\
\hline $\begin{array}{l}(\mathrm{d} 2.1 . \mathrm{i}) \\
(\mathrm{d} 2.1 . \mathrm{ii}) \\
\end{array}$ & $\begin{array}{l}d\left(v_{1}, v_{0}\right) \leq d\left(v_{2}, v_{0}\right) \\
d\left(v_{1}, v_{0}\right) \geq d\left(v_{2}, v_{0}\right)\end{array}$ & $\begin{array}{c}\left(m, d\left(v_{1}, v_{2}\right)-m, 2-d\left(v_{1}, v_{2}\right)\right) \\
\left(d\left(v_{1}, v_{2}\right)-m^{\prime}, m^{\prime}, 2-d\left(v_{1}, v_{2}\right)\right)\end{array}$ \\
\hline$(\mathrm{d} 2.2)$ & \multicolumn{2}{|c|}{$d\left(v_{1}, v_{2}\right) \leq 2-d\left(v_{0}, v_{3}\right) \leq 4-\sqrt{2}-\sqrt{2+\sqrt{2}}$} \\
\hline $\begin{array}{l}(\mathrm{d} 2.2 . \mathrm{i}) \\
(\mathrm{d} 2.2 . \mathrm{ii})\end{array}$ & $\begin{array}{l}d\left(v_{0}, v_{2}\right) \geq 0.4 \\
d\left(v_{0}, v_{2}\right) \leq 0.4\end{array}$ & $\begin{array}{l}\left(1-d\left(v_{0}, v_{3}\right) / 2,1-d\left(v_{0}, v_{3}\right) / 2, d\left(v_{0}, v_{3}\right)\right) \\
\left(1-d\left(v_{0}, v_{3}\right) / 2,1-d\left(v_{0}, v_{3}\right) / 2, d\left(v_{0}, v_{3}\right)\right)\end{array}$ \\
\hline
\end{tabular}

Table 2: Different allocations for the planar Euclidean radius game

Proposition 7.3 If the minimal circle enclosing $V$ is determined by three points $\left\{v_{1}, v_{2}, v_{3}\right\}$, and $v_{0}$ is inside this circle, then $C\left(N, v_{I I}\right) \neq \emptyset$.

Let $m=\min \left(d\left(v_{1}, v_{0}\right), d\left(v_{1}, v_{2}\right) / 2\right)$ and $m^{\prime}=\min \left(d\left(v_{2}, v_{0}\right), d\left(v_{1}, v_{2}\right) / 2\right)$. Table 2 shows allocations in $C\left(N, v_{I I}\right)$ depending on the different cases.

\section{Proof.}

We consider the subgame with the player set $\left\{v_{1}, v_{2}, v_{3}\right\}$. In the proof we distinguish between two cases depending on the angles that the three points form with the center of the circle. We assume without loss of generality that the minimal enclosing circle is of unit length, i.e., $r^{*}=1$.

Case I: $d\left(v_{i}, v_{j}\right) \geq \sqrt{2}, i, j=1,2,3, i \neq j$.

In this case the angle that the center of the minimal circle forms with each one of the pairs of the three points is at least $\pi / 2$. See Figure 7 for a graphical description of this situation. A lower bound on the length of each edge connecting such a pair is $\sqrt{2}$. In fact, a lower bound on the longer of the above 3 edges is $\sqrt{3}$, since the largest of the respective 3 angles is at least $2 \pi / 3$. In this case we also have $d\left(v_{2}, v_{0}\right)+d\left(v_{3}, v_{0}\right) \geq d\left(v_{3}, v_{2}\right) \geq \sqrt{2}$. Since 


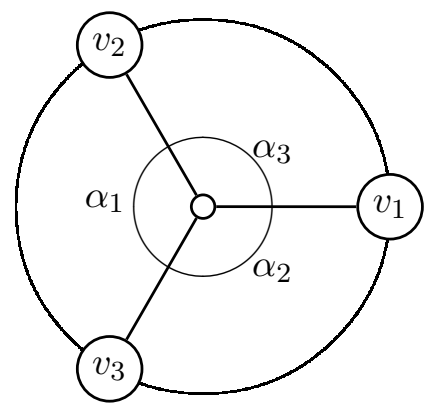

Figure 7: Three points as in Case I of Proposition $7.3, \alpha_{j} \geq \pi / 2, j=1,2,3$.

by assumption, $d\left(v_{2}, v_{0}\right) \geq d\left(v_{3}, v_{0}\right)$, we obtain $d\left(v_{2}, v_{0}\right) \geq \sqrt{2} / 2$. To conclude, in this case we have

$$
d\left(v_{1}, v_{0}\right) \geq 1, \quad d\left(v_{2}, v_{0}\right) \geq \frac{\sqrt{2}}{2}, \quad d\left(v_{i}, v_{j}\right) \geq \sqrt{2}, i, j=1,2,3, i \neq j .
$$

We consider two subcases:

Subcase (a): $d\left(v_{3}, v_{0}\right) \geq(2-\sqrt{2})$.

In this subcase, since $v_{I I}(S) \geq \sqrt{2}$, when $|S|=2$, it is sufficient to show that there is a solution to the system $x_{1}+x_{2}+x_{3}=2, x_{i}+x_{j} \leq \sqrt{2}, i, j=1,2,3, i \neq j, 0 \leq x_{1} \leq 1,0 \leq x_{2} \leq$ $\sqrt{2} / 2$, and $0 \leq x_{3} \leq(2-\sqrt{2})$. One solution is given by $x_{1}=(2 \sqrt{2}-2), x_{2}=x_{3}=(2-\sqrt{2})$. Subcase (b): $d\left(v_{3}, v_{0}\right) \leq(2-\sqrt{2})$.

We will show that in this subcase, i.e., when $d\left(v_{3}, v_{0}\right)$ is "small", there is a core allocation where $x_{3}=d\left(v_{3}, v_{0}\right)$. Specifically, we will prove that the allocation $\left(1-d\left(v_{3}, v_{0}\right) / 2,1-\right.$ $\left.d\left(v_{3}, v_{0}\right) / 2, d\left(v_{3}, v_{0}\right)\right)$ is in the core.

First, since for $i=1,2$,

$$
\sqrt{2}-d\left(v_{3}, v_{0}\right) \leq d\left(v_{i}, v_{3}\right)-d\left(v_{3}, v_{0}\right) \leq d\left(v_{i}, v_{0}\right), i=1,2,
$$

it will suffice to show that $x_{i}=1-d\left(v_{3}, v_{0}\right) / 2 \leq \sqrt{2}-d\left(v_{3}, v_{0}\right)$.

Similarly, since

$$
\sqrt{2} \leq \min \left(d\left(v_{1}, v_{3}\right), d\left(v_{2}, v_{3}\right)\right) \leq \min \left(v_{I I}\left(\left\{v_{1}, v_{3}\right\}\right), v_{I I}\left(\left\{v_{2}, v_{3}\right)\right\}\right)
$$

it will suffice to show that $x_{1}+x_{3}=x_{1}+x_{2}=1+d\left(v_{3}, v_{0}\right) / 2 \leq \sqrt{2}$.

Thus, to satisfy the above we need to show that $d\left(v_{3}, v_{0}\right) \leq 2(\sqrt{2}-1)$. Indeed, using the condition in this subcase, we obtain $d\left(v_{3}, v_{0}\right) \leq(2-\sqrt{2})<2(\sqrt{2}-1)$.

It remains to show that $x_{1}+x_{2}=2-d\left(v_{3}, v_{0}\right) \leq v_{I I}\left(\left\{v_{1}, v_{2}\right\}\right)$. Indeed, the above follows 
from Lemma 7.2 by setting $v_{i}^{\prime}=v_{i}$, for $i=1,2,3$.

We have now concluded Case I.

Case II: $d\left(v_{1}, v_{2}\right)<\sqrt{2}, d\left(v_{1}, v_{3}\right), d\left(v_{2}, v_{3}\right) \geq \sqrt{2}$.

In the second case there is (exactly) one angle that the center of the minimal circle forms with one of the pairs of the 3 points which is smaller than $\pi / 2$. Therefore, the largest angle is at least $(2 \pi-\pi / 2) / 2=3 \pi / 4$, and the second largest is at least $\pi / 2$. Without loss of generality, suppose that $d\left(v_{3}, v_{1}\right) \geq d\left(v_{3}, v_{2}\right)>d\left(v_{1}, v_{2}\right)$. (We do not assume anymore that $d\left(v_{0}, v_{1}\right) \geq d\left(v_{0}, v_{2}\right)>d\left(v_{0}, v_{3}\right)$.) Therefore,

$$
\begin{array}{r}
d\left(v_{3}, v_{1}\right) \geq \sqrt{2+\sqrt{2}}, \quad d\left(v_{1}, v_{2}\right)<\sqrt{2}, \quad d\left(v_{3}, v_{2}\right) \geq \sqrt{4-\left(d\left(v_{1}, v_{2}\right)\right)^{2}} \geq \sqrt{2} \\
\sqrt{4-\left(d\left(v_{1}, v_{2}\right)^{2}\right.} \geq 2-\frac{d\left(v_{1}, v_{2}\right)}{2}, \quad \text { whenever } d\left(v_{1}, v_{2}\right) \leq 8 / 5=1.6
\end{array}
$$

We introduce a reference system of coordinates with the origin $(0,0)$ at $C$, the center of the ball spanned by $v_{1}, v_{2}, v_{3}$. Then, $v_{1}=(-\cos \alpha,-\sin \alpha), v_{2}=(\cos \alpha,-\sin \alpha)$ and $v_{3}=(\cos \beta, \sin \beta)$ with $\pi / 4<\alpha<\beta \leq \pi / 2$, as depicted in Figure 8.

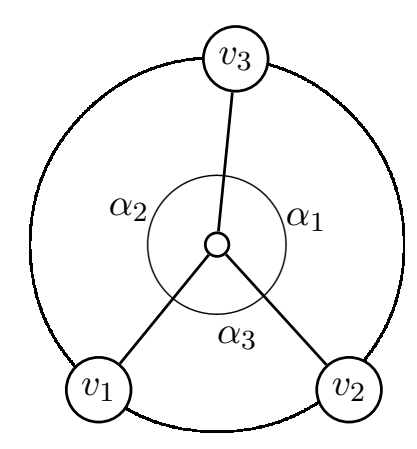

Figure 8: Three points as in Case II of Proposition 7.3, with $\alpha_{3}<\pi / 2$ and $\alpha_{1}, \alpha_{2} \geq \pi / 2$.

Define $d\left(v_{1}, v_{2}\right) / 2=a=\cos \alpha$. We first prove several useful inequalities.

For any feasible $v_{3}(\beta)=(\cos \beta, \sin \beta), \pi / 2 \geq \beta>\alpha \geq \pi / 4$,

$$
\begin{gathered}
d\left(v_{1}, v_{3}(\beta)\right) \geq \sqrt{2+2 \sin \alpha} \geq \sqrt{2+2 \sqrt{2}} \geq d\left(v_{2}, v_{3}(\beta)\right) \\
\geq \sqrt{4-\left(d\left(v_{1}, v_{2}\right)\right)^{2}} \geq \sqrt{2}>d\left(v_{1}, v_{2}\right), \\
d\left(\left(v_{1}+v_{2}\right) / 2, v_{3}(\beta)\right)=\sqrt{1+\sin ^{2} \alpha+2 \sin \beta \sin \alpha} \geq \sqrt{1+3 \sin ^{2} \alpha}
\end{gathered}
$$




$$
\begin{aligned}
& >\sqrt{5 / 2} \geq 2 a, \\
d\left(\left(v_{2}+v_{3}(\beta)\right) / 2, v_{1}\right) & =\frac{1}{2} \sqrt{2+8 \cos ^{2} \alpha+6 \cos \beta \cos \alpha+2 \sin \beta \sin \alpha} \\
& \geq \frac{1}{2} \sqrt{2+8 \cos ^{2} \alpha+2 \sin ^{2} \alpha}>1>a .
\end{aligned}
$$

We also have

$$
d\left(\left(v_{1}+v_{3}(\beta)\right) / 2, v_{2}\right)=\frac{1}{2} \sqrt{2+8 \cos ^{2} \alpha-6 \cos \beta \cos \alpha+2 \sin \beta \sin \alpha} .
$$

Since,

$$
-6 \cos \beta \cos \alpha+2 \sin \alpha \sin \beta \geq 2 \sin ^{2} \alpha-6 \cos ^{2} \alpha,
$$

we conclude that

$$
d\left(\left(v_{1}+v_{3}(\beta)\right) / 2, v_{2}\right) \geq \frac{1}{2} \sqrt{2+2 \cos ^{2} \alpha+2 \sin ^{2} \alpha} \geq 1>a .
$$

We consider two subcases.

Subcase $(\mathbf{c}): d\left(v_{3}, v_{0}\right) \leq(\sqrt{2}+\sqrt{2+\sqrt{2}}-2)$.

In this subcase we claim that the allocation

$$
\left(1-\gamma d\left(v_{3}, v_{0}\right), 1-(1-\gamma) d\left(v_{3}, v_{0}\right), d\left(v_{3}, v_{0}\right)\right)
$$

where $\gamma=(\sqrt{2}-1) /(\sqrt{2}+\sqrt{2+\sqrt{2}}-2)<1$, is in the core.

First, setting $v_{i}^{\prime}=v_{i}$, for $i=1,2,3$, and applying Lemma 7.2, we note that in this case we have $x_{1}+x_{2}=2-d\left(v_{3}, v_{0}\right) \leq v_{I I}\left(v_{1}, v_{2}\right)$.

Thus, it is sufficient to show that

$$
\begin{gathered}
x_{1}=1-\gamma d\left(v_{3}, v_{0}\right) \leq \sqrt{2+\sqrt{2}}-d\left(v_{3}, v_{0}\right) \leq d\left(v_{1}, v_{3}\right)-d\left(v_{3}, v_{0}\right) \leq d\left(v_{1}, v_{0}\right), \\
x_{1}+x_{3}=1+(1-\gamma) d\left(v_{3}, v_{0}\right) \leq \sqrt{2+\sqrt{2}} \leq d\left(v_{1}, v_{3}\right),
\end{gathered}
$$

and

$$
\begin{gathered}
x_{2}=1-(1-\gamma) d\left(v_{3}, v_{0}\right) \leq \sqrt{2}-d\left(v_{3}, v_{0}\right) \leq d\left(v_{2}, v_{3}\right)-d\left(v_{3}, v_{0}\right) \leq d\left(v_{2}, v_{0}\right), \\
x_{2}+x_{3}=1+\gamma d\left(v_{3}, v_{0}\right) \leq \sqrt{2} \leq d\left(v_{2}, v_{3}\right) .
\end{gathered}
$$


The first two conditions are satisfied whenever,

$$
d\left(v_{3}, v_{0}\right) \leq(\sqrt{2+\sqrt{2}}-1) /(1-\gamma)=(\sqrt{2}+\sqrt{2+\sqrt{2}}-2) .
$$

Similarly, the last two conditions are satisfied whenever,

$$
d\left(v_{3}, v_{0}\right) \leq(\sqrt{2}-1) / \gamma=(\sqrt{2}+\sqrt{2+\sqrt{2}}-2)
$$

Indeed, this is the condition in Subcase (c).

Subcase $(\mathbf{d}): d\left(v_{3}, v_{0}\right) \geq(\sqrt{2}+\sqrt{2+\sqrt{2}}-2)$

Subsubcase $(\mathbf{d} \mathbf{1}): d\left(v_{3}, v_{0}\right) \geq d\left(v_{3},\left(v_{1}+v_{2}\right) / 2\right)-a$.

(d1.i): $d\left(v_{1}, v_{0}\right) \leq d\left(v_{2}, v_{0}\right)$.

We show that the allocation $(m, 2 a-m, 2-2 a)$, where $m=\min \left(a, d\left(v_{1}, v_{0}\right)\right)$ is in the core. (Recall that $a=d\left(v_{1}, v_{2}\right) / 2$, as defined in page 30.) Indeed, the inequalities defining the core are satisfied:

- $x_{1}+x_{2}=2 a=d\left(v_{1}, v_{2}\right) \leq v_{I I}\left(\left\{v_{1}, v_{2}\right\}\right)$.

- $x_{1}=m \leq d\left(v_{1}, v_{0}\right)$.

- $x_{2}=2 a-m$. Hence, it suffices to prove that $2 a \leq m+d\left(v_{2}, v_{0}\right)$. If $m=a$, we have $a \leq d\left(v_{0}, v_{1}\right)$ and under our assumption, $a \leq d\left(v_{0}, v_{1}\right) \leq d\left(v_{0}, v_{2}\right)$.

When $m=d\left(v_{0}, v_{1}\right)$ we need to prove that $2 a=d\left(v_{1}, v_{2}\right) \leq d\left(v_{0}, v_{1}\right)+d\left(v_{0}, v_{2}\right)$. The latter clearly holds by the triangle inequality.

- $x_{3}=2-2 a$, and we have to prove that $2-2 a \leq d\left(v_{0}, v_{3}\right)$. Now, since $d\left(v_{0}, v_{3}\right) \geq$ $d\left(v_{3},\left(v_{1}+v_{2}\right) / 2\right)-a$, it is sufficient to verify that $d\left(v_{3},\left(v_{1}+v_{2}\right) / 2\right)-a \geq 2-2 a$, or equivalently that $d\left(v_{3},\left(v_{1}+v_{2}\right) / 2\right)+a \geq 2$.

Indeed, using the inequality (5), we obtain that:

$d\left(v_{3}, \frac{v_{1}+v_{2}}{2}\right)+a=d\left(v_{3}, \frac{v_{1}+v_{2}}{2}\right)+d\left(\frac{v_{1}+v_{2}}{2}, v_{1}\right) \geq d\left(v_{3}, c\right)+d\left(v_{1}, c\right), \forall c \in\left[\frac{v_{1}+v_{2}}{2}, C\right]$,

where $C=(0,0)$ is the center of the circle spanning $v_{1}, v_{2}, v_{3}$.

Applying the inequality for $c=C$, we obtain $d\left(v_{3},\left(v_{1}+v_{2}\right) / 2\right)+a \geq 2$.

- $x_{1}+x_{3}=2-2 a+m$. It is sufficient to prove that $2-(2 a-m) \leq d\left(v_{1}, v_{3}\right) \leq v_{I I}\left(\left\{v_{1}, v_{3}\right\}\right)$.

We prove that $x_{1}+x_{3}=2-2 a+m \leq d\left(v_{1}, v_{3}\right)$. Moreover, since $d\left(v_{1}, v_{3}\right) \geq d\left(v_{1},(0,1)\right)=$ $\sqrt{2+2 \sin \alpha}$, it will suffice to show that

$$
2-2 a+m \leq 2-a=2-\cos \alpha \leq \sqrt{2+2 \sin \alpha} .
$$


Equivalently, we will show that for $\pi / 4 \leq \alpha \leq \pi / 2, f(\alpha)=\cos \alpha+\sqrt{2+2 \sin \alpha} \geq 2$.

Define the function $g(x)=\sqrt{1-x^{2}}+\sqrt{2+2 x}$, for $\sqrt{2} / 2 \leq x \leq 1$. Since $g(x)$ is concave its minimum is given by $\min (g(\sqrt{2} / 2), g(1))=g(1)=2$.

- $x_{2}+x_{3}=2-m$.

Suppose first that $m=a$. In this case we prove that $2-\cos \alpha \leq d\left(v_{2}, v_{3}\right) \leq v_{I I}\left(\left\{v_{2}, v_{3}\right\}\right)$.

Let $u_{2}=(\cos \alpha, \sin \alpha)$. Since $d\left(v_{2}, v_{3}\right) \geq d\left(u_{2}, v_{2}\right)=2 \sin \alpha$, it will suffice to show that

$$
2-a=2-\cos \alpha \leq 2 \sin \alpha
$$

Equivalently we will show that for $\pi / 4 \leq \alpha \leq \pi / 2, f(\alpha)=\cos \alpha+2 \sin \alpha \geq 2$.

Define the function $g(x)=\sqrt{1-x^{2}}+2 x$, for $\sqrt{2} / 2 \leq x \leq 1$. Since $g(x)$ is concave its minimum is given by $\min (g(\sqrt{2} / 2), g(1))=g(1)=2$.

Next, suppose that $m=d\left(v_{1}, v_{0}\right)$. We apply Lemma 7.2 with $v_{1}^{\prime}=v_{2}, v_{2}^{\prime}=v_{3}, v_{3}^{\prime}=v_{1}$ to obtain

$$
x_{2}+x_{3}=2-d\left(v_{1}, v_{0}\right) \leq v_{I I}\left(\left\{v_{2}, v_{3}\right\}\right) .
$$

(d1.ii): $d\left(v_{1}, v_{0}\right) \geq d\left(v_{2}, v_{0}\right)$.

We show that the allocation $\left(2 a-m^{\prime}, m^{\prime}, 2-2 a\right)$, where $m^{\prime}=\min \left(a, d\left(v_{2}, v_{0}\right)\right)$ is in the core. Indeed, the core inequalities are satisfied:

- $x_{1}+x_{2}=2 a=d\left(v_{1}, v_{2}\right) \leq v_{I I}\left(\left\{v_{1}, v_{2}\right\}\right)$.

- $x_{2}=m^{\prime} \leq d\left(v_{2}, v_{0}\right)$.

- $x_{1}=2 a-m^{\prime}$. Hence, it suffices to prove that $2 a \leq m^{\prime}+d\left(v_{1}, v_{0}\right)$. If $m^{\prime}=a$, we have $a \leq d\left(v_{0}, v_{2}\right)$ and under our assumption, $a \leq d\left(v_{0}, v_{2}\right) \leq d\left(v_{0}, v_{1}\right)$.

When $m^{\prime}=d\left(v_{0}, v_{2}\right)$ we need to prove that $2 a=d\left(v_{1}, v_{2}\right) \leq d\left(v_{0}, v_{1}\right)+d\left(v_{0}, v_{2}\right)$. The latter clearly holds by the triangle inequality.

- $x_{3}=2-2 a$, and we have to prove that $2-2 a \leq d\left(v_{0}, v_{3}\right)$. The proof is the same as in the previous case (d1.i).

- $x_{2}+x_{3}=2-2 a+m^{\prime}$, and it is sufficient to prove that $2-\left(2 a-m^{\prime}\right) \leq 2-a \leq d\left(v_{2}, v_{3}\right) \leq$ $v_{I I}\left(\left\{v_{2}, v_{3}\right\}\right)$.

Indeed, the inequality $2-a \leq d\left(v_{2}, v_{3}\right)$ is proven for the respective item in (d1.i). 
- $x_{1}+x_{3}=2-m^{\prime}$. Suppose first that $m^{\prime}=d\left(v_{2}, v_{0}\right)$. In this case we apply Lemma 7.2 with $v_{1}^{\prime}=v_{1}, v_{2}^{\prime}=v_{3}, v_{3}^{\prime}=v_{2}$ to obtain

$$
x_{1}+x_{3}=2-m^{\prime} \leq v_{I I}\left(\left\{v_{1}, v_{3}\right\}\right) .
$$

Next suppose that $m^{\prime}=a$. It is sufficient to prove that $2-a \leq d\left(v_{1},(0,1)\right) \leq d\left(v_{1}, v_{3}\right) \leq$ $v_{I I}\left(\left\{v_{1}, v_{3}\right\}\right)$.

Indeed, these inequalities are proven for the respective item in (d1.i).

Subsubcase (d2): $d\left(v_{3},\left(v_{1}+v_{2}\right) / 2\right)-a \geq d\left(v_{3}, v_{0}\right) \geq \sqrt{2}+\sqrt{2+\sqrt{2}}-2$.

We will first prove the following inequality:

$$
d\left(v_{1}, v_{3}\right)-d\left(v_{0}, v_{3}\right) / 2 \geq 1
$$

The left hand side of inequality (13) satisfies:

$$
\begin{aligned}
d\left(v_{1}, v_{3}\right)-\frac{d\left(v_{0}, v_{3}\right)}{2} \geq & \sqrt{(\cos \beta+\cos \alpha)^{2}+(\sin \beta+\sin \alpha)^{2}} \\
& -\left(\sqrt{\cos ^{2} \beta+(\sin \beta+\sin \alpha)^{2}}-\cos \alpha\right) / 2 \\
= & \sqrt{2} \sqrt{1+\cos (\beta-\alpha)} \\
& -1 / 2 \sqrt{1+\sin ^{2} \alpha+2 \sin \beta \sin \alpha}+1 / 2 \cos \alpha \\
\geq & \sqrt{2} \sqrt{1+\sin \alpha}-1 / 2 \sqrt{1+\sin ^{2} \alpha+2 \sin \alpha} \\
& +1 / 2 \cos \alpha .
\end{aligned}
$$

The last inequality follows from the fact that the respective expression is a monotone decreasing function of $\beta$, for any fixed $\alpha$. Define the function $f(x)=\sqrt{2} \sqrt{1+x}-(1+x) / 2+$ $\sqrt{1-x^{2}} / 2$. (Note that the right hand side of (14) is $f(\sin \alpha)$.) The function $f(x)$ is concave and therefore its minimum is attained at one extreme point of the interval $\sqrt{2} / 2 \leq x \leq 1$. Evaluating, we obtain: $f(\sqrt{2} / 2)=1 / 2 \sqrt{2} \sqrt{4+2 \sqrt{2}}-1 / 2=1.3477$ and $f(1)=1$. Therefore, inequality (13) holds.

(d2.1): $d\left(v_{1}, v_{2}\right) \geq 2-d\left(v_{0}, v_{3}\right)$.

(Note that in Subcase $(\mathrm{d})$, since $d\left(v_{0}, v_{3}\right) \geq \sqrt{2}+\sqrt{2+\sqrt{2}}-2$, the above is satisfied if $\left.d\left(v_{1}, v_{2}\right) \geq 4-\sqrt{2}-\sqrt{2+\sqrt{2}}\right)$.

(d2.1.i): $d\left(v_{1}, v_{0}\right) \leq d\left(v_{2}, v_{0}\right)$.

Set $x_{1}=m, x_{2}=d\left(v_{1}, v_{2}\right)-m, x_{3}=2-d\left(v_{1}, v_{2}\right)$, where $m=\min \left(d\left(v_{1}, v_{0}\right), d\left(v_{1}, v_{2}\right) / 2\right)$. We will show that this allocation is in the core. 
We have $x_{1} \leq d\left(v_{1}, v_{0}\right)$. Also, if $x_{1}=d\left(v_{1}, v_{2}\right) / 2$, then $x_{2}=d\left(v_{1}, v_{2}\right) / 2 \leq d\left(v_{1}, v_{0}\right) \leq$ $d\left(v_{2}, v_{0}\right)$. Otherwise, from the triangle inequality $x_{2}=d\left(v_{1}, v_{2}\right)-d\left(v_{1}, v_{0}\right) \leq d\left(v_{2}, v_{0}\right)$. Also, from (d2.1) we obtain $x_{3} \leq d\left(v_{3}, v_{0}\right)$.

We have $x_{1}+x_{2}=d\left(v_{1}, v_{2}\right) \leq v_{I I}\left(\left\{v_{1}, v_{2}\right\}\right)$.

Suppose first that $x_{1}=d\left(v_{1}, v_{2}\right) / 2$. Then, $x_{1}+x_{3}=x_{2}+x_{3}=2-d\left(v_{1}, v_{2}\right) / 2$. Since $d\left(v_{2}, v_{3}\right) \leq d\left(v_{1}, v_{3}\right)$, and $d\left(v_{j}, v_{3}\right) \leq v_{I I}\left(\left\{v_{j}, v_{3}\right\}\right)$, for $j=1,2$, it will suffice to show that $2-d\left(v_{1}, v_{2}\right) / 2 \leq d\left(v_{2}, v_{3}\right)$.

If $x_{1}=d\left(v_{1}, v_{0}\right)$, then $x_{2}+x_{3}=2-d\left(v_{1}, v_{0}\right)$. Applying Lemma 7.2 with $v_{1}^{\prime}=v_{2}, v_{2}^{\prime}=v_{3}$ and $v_{3}^{\prime}=v_{1}$, we obtain $x_{2}+x_{3} \leq v_{I I}\left(\left\{v_{2}, v_{3}\right\}\right)$. In this case we have $x_{1}+x_{3} \leq d\left(v_{1}, v_{2}\right) / 2+$ $2-d\left(v_{1}, v_{2}\right)=2-d\left(v_{1}, v_{2}\right) / 2$. Again, it will suffice to show that $2-d\left(v_{1}, v_{2}\right) / 2 \leq d\left(v_{2}, v_{3}\right)$.

Indeed, the latter holds since applying inequalities $(7), d\left(v_{2}, v_{3}\right) \geq \sqrt{4-\left(d\left(v_{1}, v_{2}\right)\right)^{2}}$ and applying inequality (8), $\sqrt{4-\left(d\left(v_{1}, v_{2}\right)\right)^{2}} \geq 2-d\left(v_{1}, v_{2}\right) / 2$, whenever $d\left(v_{1}, v_{2}\right) \leq 8 / 5=1.6$. (Recall that in Case II $d\left(v_{1}, v_{2}\right)<\sqrt{2}$.)

(d2.1.ii): $d\left(v_{1}, v_{0}\right) \geq d\left(v_{2}, v_{0}\right)$.

Set $x_{2}=m^{\prime}, x_{1}=d\left(v_{1}, v_{2}\right)-m^{\prime}, x_{3}=2-d\left(v_{1}, v_{2}\right)$, where $m^{\prime}=\min \left(d\left(v_{2}, v_{0}\right), d\left(v_{1}, v_{2}\right) / 2\right)$. We will show that this allocation is in the core.

Then, $x_{2} \leq d\left(v_{1}, v_{0}\right)$. Also, if $x_{2}=d\left(v_{1}, v_{2}\right) / 2$, then $x_{1}=d\left(v_{1}, v_{2}\right) / 2 \leq d\left(v_{2}, v_{0}\right) \leq$ $d\left(v_{1}, v_{0}\right)$. Otherwise, from the triangle inequality $x_{1}=d\left(v_{1}, v_{2}\right)-d\left(v_{2}, v_{0}\right) \leq d\left(v_{1}, v_{0}\right)$. Also, from (d2.1) we obtain $x_{3} \leq d\left(v_{3}, v_{0}\right)$.

We have $x_{1}+x_{2}=d\left(v_{1}, v_{2}\right) \leq v_{I I}\left(\left\{v_{1}, v_{2}\right\}\right)$.

Suppose first that $x_{2}=d\left(v_{1}, v_{2}\right) / 2$. Then, $x_{1}+x_{3}=x_{2}+x_{3}=2-d\left(v_{1}, v_{2}\right) / 2$. Since $d\left(v_{2}, v_{3}\right) \leq d\left(v_{1}, v_{3}\right)$, and $d\left(v_{j}, v_{3}\right) \leq v_{I I}\left(\left\{v_{j}, v_{3}\right\}\right)$ for $j=1,2$, it will suffice to show that $2-d\left(v_{1}, v_{2}\right) / 2 \leq d\left(v_{2}, v_{3}\right)$.

If $x_{2}=d\left(v_{2}, v_{0}\right)$, then $x_{1}+x_{3}=2-d\left(v_{1}, v_{0}\right)$. Applying Lemma 7.2 with $v_{1}^{\prime}=v_{1}, v_{2}^{\prime}=v_{3}$, and $v_{3}^{\prime}=v_{2}$, we obtain $x_{2}+x_{3} \leq v_{I I}\left(\left\{v_{2}, v_{3}\right\}\right)$. In this case we have $x_{2}+x_{3} \leq d\left(v_{1}, v_{2}\right) / 2+$ $2-d\left(v_{1}, v_{2}\right)=2-d\left(v_{1}, v_{2}\right) / 2$. Again, it will suffice to show that $2-d\left(v_{1}, v_{2}\right) / 2 \leq d\left(v_{2}, v_{3}\right)$.

Indeed, the latter holds since applying first inequality (7) and then (8), it holds $d\left(v_{2}, v_{3}\right) \geq$ $\sqrt{4-\left(d\left(v_{1}, v_{2}\right)\right)^{2}} \geq 2-d\left(v_{1}, v_{2}\right) / 2$, whenever $d\left(v_{1}, v_{2}\right) \leq 8 / 5=1.6$.

(d2.2): $d\left(v_{1}, v_{2}\right) \leq 2-d\left(v_{0}, v_{3}\right) \leq 4-\sqrt{2}-\sqrt{2+\sqrt{2}}$.

(d2.2.i): $d\left(v_{0}, v_{2}\right) \geq 0.4$.

We prove that the allocation $\left(1-d\left(v_{0}, v_{3}\right) / 2,1-d\left(v_{0}, v_{3}\right) / 2, d\left(v_{0}, v_{3}\right)\right)$ is in the core. The inequalities defining the core are:

- $x_{1}=1-d\left(v_{0}, v_{3}\right) / 2 \leq d\left(v_{1}, v_{0}\right)$. Thus, we have to prove that $1 \leq d\left(v_{1}, v_{0}\right)+d\left(v_{0}, v_{3}\right) / 2$.

From the triangle inequality and inequality (13) we have $d\left(v_{1}, v_{0}\right)+d\left(v_{0}, v_{3}\right) / 2 \geq$ $d\left(v_{1}, v_{3}\right)-d\left(v_{0}, v_{3}\right) / 2 \geq 1$.

- $x_{2}=1-d\left(v_{3}, v_{0}\right) / 2 \leq d\left(v_{2}, v_{0}\right)$. Indeed, $x_{2}=1-d\left(v_{3}, v_{0}\right) / 2<1-1.2 / 2=0.4 \leq$ $d\left(v_{2}, v_{0}\right)$. 
- The inequality $x_{1}+x_{2} \leq v_{I I}\left(\left\{v_{1}, v_{2}\right\}\right)$ follows from Lemma 7.2 , setting $v_{i}^{\prime}=v_{i}$, for $i=1,2,3$.

- $x_{1}+x_{3}=1+d\left(v_{0}, v_{3}\right) / 2$. From inequality (13) we obtain $1+d\left(v_{0}, v_{3}\right) / 2 \leq d\left(v_{1}, v_{3}\right)$. Thus, since $d\left(v_{1}, v_{3}\right) \leq v_{I I}\left(\left\{v_{1}, v_{3}\right\}\right)$, we have $x_{1}+x_{3} \leq v_{I I}\left(\left\{v_{1}, v_{3}\right\}\right)$.

- Finally, we have to prove the inequality $x_{2}+x_{3}=1+d\left(v_{0}, v_{3}\right) / 2 \leq v_{I I}\left(\left\{v_{2}, v_{3}\right\}\right)$.

Using (d2.2) we obtain

$$
1+d\left(v_{0}, v_{3}\right) / 2 \leq 1+\left(2-d\left(v_{1}, v_{2}\right)\right) / 2=2-d\left(v_{1}, v_{2}\right) / 2 .
$$

We showed above in the previous case that $2-d\left(v_{1}, v_{2}\right) / 2 \leq d\left(v_{2}, v_{3}\right)$. Hence, $x_{2}+x_{3} \leq$ $d\left(v_{2}, v_{3}\right) \leq v_{I I}\left(\left\{v_{2}, v_{3}\right\}\right)$.

(d2.2.ii): $d\left(v_{2}, v_{0}\right) \leq 0.4$.

Recall that in $(\mathrm{d} 2.2)$ we already have $d\left(v_{1}, v_{2}\right) / 2 \leq\left(2-d\left(v_{0}, v_{3}\right)\right) / 2 \leq 2-(\sqrt{2}+$ $\sqrt{2+\sqrt{2}}) / 2$.

Again, like in the previous case, we prove that the allocation $\left(1-d\left(v_{0}, v_{3}\right) / 2,1-d\left(v_{0}, v_{3}\right) / 2\right.$, $\left.d\left(v_{0}, v_{3}\right)\right)$ is in the core. Indeed, the inequalities that define the core are:

- $x_{1}=1-d\left(v_{0}, v_{3}\right) / 2 \leq d\left(v_{1}, v_{0}\right)$. Again, from the triangle inequality and equations (13) and (14) we have $d\left(v_{1}, v_{0}\right)+d\left(v_{0}, v_{3}\right) / 2 \geq d\left(v_{1}, v_{3}\right)-d\left(v_{0}, v_{3}\right) / 2 \geq 1$.

- $x_{2}=1-d\left(v_{3}, v_{0}\right) / 2 \leq d\left(v_{2}, v_{0}\right)$.

Here we will use the fact that $a=\cos \alpha=d\left(v_{1}, v_{2}\right) / 2 \leq 2-(\sqrt{2}+\sqrt{2+\sqrt{2}}) / 2<0.6$, and use the function

$$
\begin{aligned}
f(x, y)= & \sqrt{(\cos y-\cos x)^{2}+(\sin y+\sin x)^{2}} \\
& -1 / 2\left(\sqrt{\cos ^{2} y+(\sin x+\sin y)^{2}}-\cos x\right), \\
& \text { with } \arccos (0.6) \leq x \leq \pi / 2, x \leq y \leq \pi / 2,
\end{aligned}
$$

Note that

$$
\begin{aligned}
f(x, y) & =\sqrt{2-2 \cos (x+y)}-1 / 2 \sqrt{1+\sin ^{2} x+2 \sin x \sin y}+1 / 2 \cos x \\
& \geq \sqrt{2-2 \cos 2 x}-1 / 2(1+\sin x)+\frac{\cos x}{2} .
\end{aligned}
$$

Consider the function

$$
g(z)=\sqrt{4-4 z^{2}}-1 / 2\left(1+\sqrt{1-z^{2}}\right)+z / 2=\left(3 \sqrt{1-z^{2}}+z-1\right) / 2,
$$


for $0 \leq z \leq 0.6$. This function is the right-hand side of the above inequality at $z=\cos x$. Clearly $g(z)$ is concave since its second derivative is $g^{\prime \prime}(z)=-3 / 2 z^{2} /\left(1-z^{2}\right)^{(3 / 2)}-$ $3 / 21 / \sqrt{1-z^{2}}$. Therefore, its minimum is attained at one of the extreme points, hence $g(z) \geq \min \{g(0), g(0.6)\}=1$.

Now, since $d\left(v_{2}, v_{3}\right)-d\left(v_{0}, v_{3}\right) / 2=f(\alpha, \beta)$, we have that $d\left(v_{2}, v_{3}\right)-d\left(v_{0}, v_{3}\right) / 2 \geq 1$, which in turn, by the triangle inequality implies

$$
x_{2}=1-d\left(v_{3}, v_{0}\right) / 2 \leq d\left(v_{2}, v_{3}\right)-d\left(v_{0}, v_{3}\right) \leq d\left(v_{2}, v_{0}\right) .
$$

- The inequality $x_{1}+x_{2}=2-d\left(v_{0}, v_{3}\right) \leq v_{I I}\left(\left\{v_{1}, v_{2}\right\}\right)$ follows from Lemma 7.2 while setting $v_{i}^{\prime}=v_{i}$, for $i=1,2,3$.

- $x_{1}+x_{3}=1+d\left(v_{0}, v_{3}\right) / 2$. From inequality (13) we obtain $1+d\left(v_{0}, v_{3}\right) / 2 \leq d\left(v_{1}, v_{3}\right)$. Thus, since $d\left(v_{1}, v_{3}\right) \leq v_{I I}\left(\left\{v_{1}, v_{3}\right\}\right)$, we have $x_{1}+x_{3} \leq v_{I I}\left(\left\{v_{1}, v_{3}\right\}\right)$.

- Finally, we have to prove the inequality $x_{2}+x_{3}=1+d\left(v_{0}, v_{3}\right) / 2 \leq v_{I I}\left(\left\{v_{2}, v_{3}\right\}\right)$. However, the proof is the same as for the previous case (d2.2.i).

Propositions 7.1, 7.2 and 7.3, all together, conclude the proof of Theorem 5.5. 